TAO, Vol. 10, No. 4, 651-692, December 1999

\title{
Experiments with a Spectral Convection Model
}

\author{
Hung-Chi Kuo ${ }^{1}$ and Chao-Tzuen Cheng ${ }^{1}$
}

(Manuscript received 25 January 1999, in final form 15 September 1999)

\begin{abstract}
A new spectral moist convection model that employs both the least assumptions in moist physics and a very accurate solution method is presented. The temperature and pressure in the model are diagnostically determined from thermodynamics. There is no need to predict water vapor and condensate separately; rather, they are diagnostically separated from the predicted total airborne water. The model allows a modular separation of dynamics and thermodynamics; the link between dynamics and thermodynamics is through the pressure gradient force. The modular separation allows the possibility of having a detailed, fine resolution, nonhydrostatic cloud model and a coarse resolution, hydrostatic model which can be run side by side with the identical moist thermodynamics. The height coordinate of the nonhydrostatic model can also extend into the hydrostatic regime. The only differences between the hydrostatic and nonhydrostatic models are spatial resolution and the way vertical motion is computed. We have performed numerical experiments in the nonhydrostatic model for acoustic adjustment and moist convection. The discontinuity in thermodynamics due to phase change is modified in the model by the "gradual saturation" technique.
\end{abstract}

(Key words: Fourier-Chebyshev spectral method, Acoustic adjustment, Lanczos filter)

\section{INTRODUCTION}

Global models have become important tools for weather and climate simulations. However, these models have simplified hydrostatic dynamics and coarse vertical and horizontal resolution, so they are unable to explicitly simulate most of the cloud patterns that are crucial to climate dynamics. Because of their important effects on radiative transfer, hydrological cycles and apparent heat sources, moist convections must be more accurately treated in climate models. The real atmosphere contains a myriad of cloud structures which modulate radiative fluxes and which modify atmospheric structure by condensing water at one level and

\footnotetext{
${ }^{1}$ Department of Atmospheric Sciences, National Taiwan University, Taipei, Taiwan, ROC
} 
reevaporating it at another level. This deep and shallow moist convection can be modeled more accurately with a nonhydrostatic model. Nonhydrostatic convection models can be based on unfiltered or filtered systems. The filtered systems include the anelastic and Boussinesq equations. Ogura and Phillips (1962) derived the anelastic system under the assumptions that the percentage range of potential temperature is small and the time scale is set by the BruntVäisälä a frequency. The anelastic equations reduce to the Boussinesq equations under the additional assumption that the vertical scale of motion is small compared with the depth of an isentropic atmosphere. In an unfiltered system, one has to cope with the propagation of acoustic waves. Often the pressure is used as a prognostic variable of the model. Since pressure is not a conservative property, the prognostic equation for pressure has been derived from other conservation laws under some approximation of moist thermodynamics, which usually involves a definition of equivalent potential temperature. This is also a problem with existing general circulation models, since they are based on the quasi-static primitive equations in sigma coordinates and have a host of thermodynamic approximations associated with the use of equivalent potential temperature, moist static energy, etc.

With progress in computers and computational techniques, we have often experienced that the return to the first principles of physics enables a model to cope more easily with the complexity of the atmosphere. Ooyama (1990) proposed a "primitive" form of moist thermodynamics. Instead of using pressure as a prognostic variable, Ooyama's model uses the conservation of the entropy density, the momentum density and the total moisture density as predicted variables. With an accurate definition of entropy density in the moist atmosphere, Ooyama's proposal involves the least assumptions in reversible moisture physics. Since the pressure field no longer is a prognostic variable, Ooyama's proposal also allows the extension of a nonhydrostatic height coordinate model into the hydrostatic regime. This extension may be useful in simulations of weather systems that involve a wide range of horizontal scales.

We have constructed a new spectral moist convection model that employs both the least assumptions in moist physics and a very accurate solution method. Our modeling effort involves the Fourier-Chebyshev spectral discretization similar to that in Kuo and Schubert (1988) and the moist thermodynamics of the "primitive" form in Ooyama (1990). We believe a sound basis for moist thermodynamics and an accurate treatment of discretization are important for the improvement of cloud modeling. The governing equations are presented in section 2 . Section 3 describes the solution method. Numerical results are covered in section 4 . Section 5 contains the concluding remarks.

\section{GOVERNING EQUATIONS}

We consider the two-dimensional $(x-z)$ case described below. The "primitive" form of moist thermodynamics makes model predictions strictly in terms of conservative properties, in particular the density of dry air $\xi$, density of total airborne moisture $\eta$, entropy density $\sigma$, the momentum densities $U=\rho u, W=\rho w$, where $\rho=\xi+\eta$. Here we have included precipitation (or drizzle) effects in $Q_{\eta}$ and diabatic (e.g., radiative) effects in $Q_{\sigma}$ in our equations. The prognostic equations for the conservative variables are 


$$
\begin{gathered}
\frac{\partial U}{\partial t}+\frac{\partial(U u)}{\partial x}+\frac{\partial(U w)}{\partial z}+\frac{\partial p}{\partial x}=0 \\
\frac{\partial W}{\partial t}+\frac{\partial(W u)}{\partial x}+\frac{\partial(W w)}{\partial z}+g \rho+\frac{\partial p}{\partial z}=0 \\
\frac{\partial \sigma}{\partial t}+\frac{\partial(\sigma u)}{\partial x}+\frac{\partial(\sigma w)}{\partial z}=Q_{\sigma} \\
\frac{\partial \xi}{\partial t}+\frac{\partial(\xi u)}{\partial x}+\frac{\partial(\xi w)}{\partial z}=0 \\
\frac{\partial \eta}{\partial t}+\frac{\partial(\eta u)}{\partial x}+\frac{\partial(\eta w)}{\partial z}=Q_{\eta}
\end{gathered}
$$

where

$$
\rho=\xi+\eta, \quad u=\frac{U}{\rho}, \quad w=\frac{W}{\rho}
$$

The above constitute eight equations for the five prognostic variables $\xi, \eta, \sigma, U, W$, and the four diagnostic variables $\rho, u, w$ and $p$. The system is closed by the thermodynamic diagnosis, the input of which is $\xi, \eta, \sigma$, and the output of which is temperature, pressure and the partition of $\eta$ into its vapor and condensate parts. This requires writing two formulas (depending on whether the total airborne moisture $\eta$ is entirely in the vapor phase or is partially condensed) for the entropy $\sigma(\xi, \eta, T)$, iteratively solving for two temperatures $\left(T_{1}\right.$ and $\left.T_{2}\right)$ and then using

$$
T=\max \left(T_{1}, T_{2}\right)
$$

If $T_{\mathrm{J}}>T_{2}$ (unsaturated) $p_{\mathrm{v}}=\eta R_{v} T, \quad \eta_{v}=\eta, \quad \eta_{c}=0$

If $T_{2}>T_{1}$ (saturated) $p_{\mathrm{v}}=E(T), \quad \eta_{v}=\eta_{*}(T), \quad \eta_{c}=\eta-\eta_{v}$

$$
p_{a}=\xi R_{a} T, p=p_{a}+p_{v}
$$


Here $\eta_{\mathrm{v}}$ and $\eta_{c}$ are the densities of vapor and condensate respectively, $p_{a}$ and $p_{v}$ the partial pressures of dry air and water vapor respectively, $E(T)$ the saturation vapor pressure and $\eta_{*}(T)=E(T) /\left(R_{v} T\right)$ the mass density of saturated vapor.

When the hydrostatic approximation is made, as described by Ooyama (1990) and DeMaria (1995), (2.2) is replaced by a simple diagnostic equation

$$
\frac{\partial}{\partial z}\left(\rho C^{2} \frac{\partial w}{\partial z}\right)=-\frac{\partial}{\partial z}\left(v_{i} \frac{\partial p}{\partial x_{i}}+\rho C^{2} \frac{\partial v_{i}}{\partial x_{i}}\right)-\frac{\partial \rho v_{i}}{\partial x_{i}} g+\frac{\partial}{\partial z}\left(\frac{\partial p}{\partial \sigma} Q_{\sigma}+\frac{\partial p}{\partial \rho} Q_{\eta}\right)+g Q_{\eta_{i}} i=1,2
$$

where

$$
C^{2}=\frac{\partial p}{\partial \rho}+\frac{\sigma}{\rho} \frac{\partial p}{\partial \sigma}
$$

is the Laplace adiabatic sound speed. Derivations of the vertical motion diagnostic equations can be found in appendix A. The replacement of (2.2) with (2.9) is the only change necessary to convert the nonhydrostatic equations to their hydrostatic form. This diagnostic equation is a one-dimensional (height) second-order elliptic equation that can be solved efficiently using a direct method. DeMaria (1995) found that hydrostatic solutions of (2.9) are very sensitive to the method used to solve the diagnostic vertical velocity equation. The sensitivity can be eliminated, as described by DeMaria (1995), by adding an extra term to the diagnostic equation that ensures the solution does not drift away from the hydrostatic balance due to numerical approximation. With the diagnostic vertical velocity equation, Ooyama's formulation allows us to design a numerical model in height coordinates that can be used in hydrostatic and nonhydrostatic regimes.

\section{SOLUTION METHODS}

The simulation of moist convection places great demands on the spatial discretization schemes used in numerical models. We will use a scheme which is spectral in both directions. We shall solve the above system of equations on the domain $0 \leq x \leq L, 0 \leq z \leq H$, with the assumption that all variables are periodic in $x$ and $W=0$ on $z=0, H$. In the $x$ direction, Fourier basis functions are used so that the periodicity is built into each basis function. In the $z$ direction, Chebyshev polynomial basis functions are used; the top and bottom boundary conditions are not satisfied by each basis function, but rather by the series as a whole. Details of the Chebyshev tau method can be found in Fulton and Schubert (1987) and Kuo and Schubert (1988). In the following we discuss the spectral method for solving the system. 
a. Fourier-Chebyshev method

The dependent variables (e.g., $\xi$ ) are approximated by the series expansions

$$
\xi(x, z, t)=\sum_{m=-M}^{M} \sum_{n=0}^{N} \hat{\xi}_{m n}(t) T_{n}\left(z^{\prime}\right) e^{2 \pi i m x / l}
$$

where the $T_{n}\left(z^{\prime}\right)$ are the Chebyshev polynomials defined on the interval $-1 \leq z^{\prime} \leq 1$ by $T_{n}\left(z^{\prime}\right)$ $=\cos (n \phi)$ with $z^{\prime}=2 z / H-1=\cos \phi$. Defining the Fourier-Chebyshev inner product of two functions $f(x, z)$ and $g(x, z)$ as

$$
\langle f, g\rangle=\frac{1}{L} \int_{-1}^{1} \int_{0}^{L} \frac{f\left(x, z^{\prime}\right) g^{*}\left(x, z^{\prime}\right)}{\left(1-z^{\prime 2}\right)^{1 / 2}} d x d z^{\prime}
$$

where the star denotes complex conjugate. The spectral coefficient $\hat{\xi}_{m i t}(t)$ is given by

$$
\hat{\xi}_{m n}(t)=\frac{2}{\pi c_{n}}\left\langle\xi(x, z, t), T_{n}\left(z^{\prime}\right) e^{2 \pi i m x / L}\right\rangle
$$

with $c_{n}=\left\{\begin{array}{ll}2 & n=0 \\ 1 & n>0\end{array}\right\}$. Equation (3.3) is the transformation from physical space to FourierChebyshev spectral space and (3.1) is the transformation back. The evaluation of (3.2) can be done by the fast Fourier transform and the fast Chebyshev transform. The total $N_{z}$ collocation points for the fast Chebyshev transform in the vertical are determined as

$$
Z_{j}=\frac{H}{2}\left[\cos \left(\frac{j \pi}{N_{z}}\right)+1\right], j=0,1,2, \ldots N_{z}
$$

With the nonlinear terms defined by,

$$
\begin{aligned}
& A=U u ; B=W u, \\
& C=U w ; D=W w, \\
& \mathrm{E}=\sigma u ; F=\sigma w, \\
& G=\xi u ; H=\xi w,
\end{aligned}
$$




$$
I=\eta u ; J=\eta w
$$

and to be computed by the transform method. To eliminate aliasing error in the quadratic nonlinear terms in the transform method, $3 M$ points in the $x$ direction and $3 N / 2$ points in the $z$ direction are needed in the physical domain. The tau equations for (2.1)-(2.5) are

$$
\begin{gathered}
\frac{d \hat{U}_{m n}}{d t}+\hat{A}_{m n}^{(1,0)}+\hat{B}_{m n}^{(0,1)}+\hat{p}_{m n}^{(1,0)}=0 \\
\frac{d \hat{W}_{m n}}{d t}+\hat{C}_{m n}^{(1,0)}+\hat{D}_{m n}^{(0,1)}+\left(\hat{\xi}_{m n}+\hat{\eta}_{m n}\right) g+\hat{p}_{m n}^{(0,1)}=0 \\
\frac{d \hat{\sigma}_{m n}}{d t}+\hat{E}_{m n}^{(1,0)}+\hat{F}_{m n}^{(0,1)}=0 \\
\frac{d \hat{\xi}_{m n}}{d t}+\hat{G}_{m n}^{(1,0)}+\hat{H}_{m n}^{(0,1)}=0 \\
\frac{d \hat{\eta}_{m n}}{d t}+\hat{I}_{m n}^{(1,0)}+\hat{J}_{m n}^{(0,1)}=0
\end{gathered}
$$

where the $x$ derivative of the spectral coefficients is denoted by the superscript $(1,0)$ and the $z$ derivative of the spectral coefficients is denoted by the superscript $(0,1)$. The time integration of (3.5) is done with the fourth-order Runge-Kutta scheme for all the modes $-M \leq m \leq-M$ and $0 \leq n \leq N$ with the exception of the spectral coefficients $\hat{W}_{m, N-1}$ and $\hat{W}_{m, N}$. According to the $\tau$ method, the last two vertical modes of $\hat{W}_{m n}$ are to be obtained from the vertical boundary conditions $(W(0)=W(H)=0)$. Namely, we solve $\hat{W}_{m, N-1}$ and $\hat{W}_{m, N}$ by

$$
\sum_{p=0}^{N}(-1)^{p} \hat{W}_{m p}=0
$$

and

$$
\sum_{p=0}^{N} \hat{W}_{m p}=0,
$$

The relation between $\hat{A}_{m n}^{(1,0)}$ and $\hat{A}_{m n}$ (the spectral coefficient of $\mathrm{A}$ ) is 


$$
\hat{A}_{m n}^{(1,0)}=i\left(\frac{2 \pi m}{L}\right) \hat{A}_{m n},
$$

while the relation between $\hat{B}_{m n}^{(0,1)}$ and $\hat{B}_{m n}$ (the spectral coefficient of $\mathrm{B}$ ) is

$$
\hat{B}_{m n}^{(0,1)}=\frac{4}{H c_{n}} \sum_{\substack{n=n+1 \\ p+n \text { odd }}}^{N} p \hat{B}_{m p} .
$$

Although the spectral evaluation of $z$ derivatives by (3.8) looks at first sight more difficult than the spectral evaluation of $x$ derivatives by (3.7), such is not the case. Equation (3.8) yields the (backward) recurrence formula

$$
c_{n-1} \hat{B}_{m, n-1}^{(0,1)}-\hat{B}_{m, n+1}^{(0,1)}=\frac{4}{H} n \hat{B}_{m, n} \quad(n=1,2, \ldots, N-1)
$$

with the starting values $\hat{B}_{m, N+1}^{(0,1)}=\hat{B}_{m, N}^{(0,1)}=0$. For fixed $m$, the use of (3.9) allows the $N$ values of $\hat{B}_{m, n}^{(0,1)}$ to be computed in $O(N)$ operations.

b. Pressure gradient across a cloud edge

The pressure field is diagnosed from the equation of state in our model. The fields of temperature and liquid water density can have discontinuities in the first derivative across a cloud edge due to phase change. With a sufficiently smooth density field, the first derivative discontinuity in the temperature field causes the first derivative discontinuity in the pressure field across a cloud edge. To avoid the Gibb's phenomena in a spectral model, we need to adapt the "graduation saturation" technique. Since the temperature and the liquid water density fields do not explicitly appear in (3.5), only treatment for the pressure field is required. The basic idea is presented in equations (3.10) and (3.11) in this section. The numerical results are presented in section 4 (i.e., Figs. 1, 2, and 3).

At any spatial point, $p=P(\xi, \eta, \sigma)$, thus we have

$$
\nabla p=P_{\xi} \nabla \xi+P_{\eta} \nabla \eta+P_{\sigma} \nabla \sigma
$$

The $P$-coefficients are known functions of $(x, h, \mathrm{~s})$ so that (3.10) for $\nabla p$ could now be used for the pressure gradient force in the momentum equations in the spectral model. However, Ooyama discusses how this can cause Gibbs' phenomena near cloud edges. As a solution he proposes weighted averages of the $P$-coefficients for saturated and unsaturated conditions. The overlap of the weighting coefficients is adjusted to the model spatial resolution. The weighting coefficients are

$$
\Omega_{1}=\frac{1}{2}\left[1+\tanh \left(\frac{T_{1}-T_{2}}{\Delta T_{12}}\right)\right]
$$




$$
\Omega_{2}=1-\Omega_{1},
$$

If the $P_{\xi}^{(1)}$ and $P_{\xi}^{(2)}$ are computed from $\mathrm{T}_{1}$ (temperature in unsaturated region) and $\mathrm{T}_{2}$ (temperature in saturated region) respectively, then the weighted average of $P_{\xi}$ across cloud edge is given by

$$
P_{\xi}=\Omega_{1} P_{\xi}^{(1)}+\Omega_{2} P_{\xi}^{(2)}
$$

Equation (3.11) can also apply to the calculations of $P_{\eta}$ and $P_{\sigma}$. The formula for $P_{\eta}^{(1)}, \mathrm{P}_{\eta}^{(2)}, P_{\xi}^{(1)}$, $P_{\xi}^{(2)}, P_{\sigma}^{(1)}$ and $P_{\sigma}^{(2)}$ can be found in Ooyama (1990). The derivation of the formula is given in appendix $\mathrm{B}$.

\section{NUMERICAL RESULTS}

a. One-dimensional experiment

Our first experiment (EXP1) is the calculation of the pressure gradient across a cloud edge in one dimension. We consider a domain of $0 \leq x \leq 2000 \mathrm{~m}$. Let

$$
f(x)=a+b \exp \left[-\left(\frac{x-x_{0}}{\Delta x}\right)^{2}\right],
$$

where $x_{0}=1000 \mathrm{~m}$ and $\Delta x=250 \mathrm{~m}$. The thermodynamic variables are specified according to (4.1) with different constants $a$ and $b$. We take $b=65 \mathrm{~m}^{2} \mathrm{~s}^{-2} \mathrm{~K}^{-1}$ and $a=220 \mathrm{~m}^{2} \mathrm{~s}^{-2} \mathrm{~K}^{-1}$ for the entropy density $\sigma$. Similarly, we take $b=0.01 \mathrm{~kg} \mathrm{~m}^{-3}, a=0.0078 \mathrm{~kg} \mathrm{~m}^{-3}$ for total water density $\eta$ and take $b=0.0095 \mathrm{~kg} \mathrm{~m}^{-3}, a=1.1135 \mathrm{~kg} \mathrm{~m}^{-3}$ for dry air density $\xi$.

The profiles of $\sigma, \xi$ and $\eta$ are shown in Fig. 1. The profiles of the diagnosed temperature $T$, pressure $p$ and condensed water density $\eta_{c}$ are shown in Fig. 2. There are discontinuities in the first derivative of the $T, p$ and $\eta_{\mathrm{c}}$ fields across the cloud edge. Figure 3 shows the pressure gradient calculated from (a) direct differentiation of the $p$ profile of Fig. $2 b$ by 48 grid points Fourier spectral method and from (b) Fourier spectral method by (3.10) and (3.11) with 48 grid points. The $\Delta T_{12}$ used here is $10^{-7} \mathrm{~K}$. The Chebyshev method yields results similar to Fig. 3 , and thus is not shown here. It is concluded from Fig. 3 that the pressure gradient calculation by (3.10) and (3.11) greatly reduces the Gibbs phenomenon in the Fourier-Chebyshev spectral method.

\section{b. Two-dimensional experiments}

For the two-dimensional experiments, we have used $L=H=2500 \mathrm{~m}, M=16, N=32$ and $\Delta t=0.075 \mathrm{~s}$ in our calculation. To improve time integration efficiency, the subgrid diffusion process in the model is handled in spectral space by applying the Lanczos filter to the tendency 

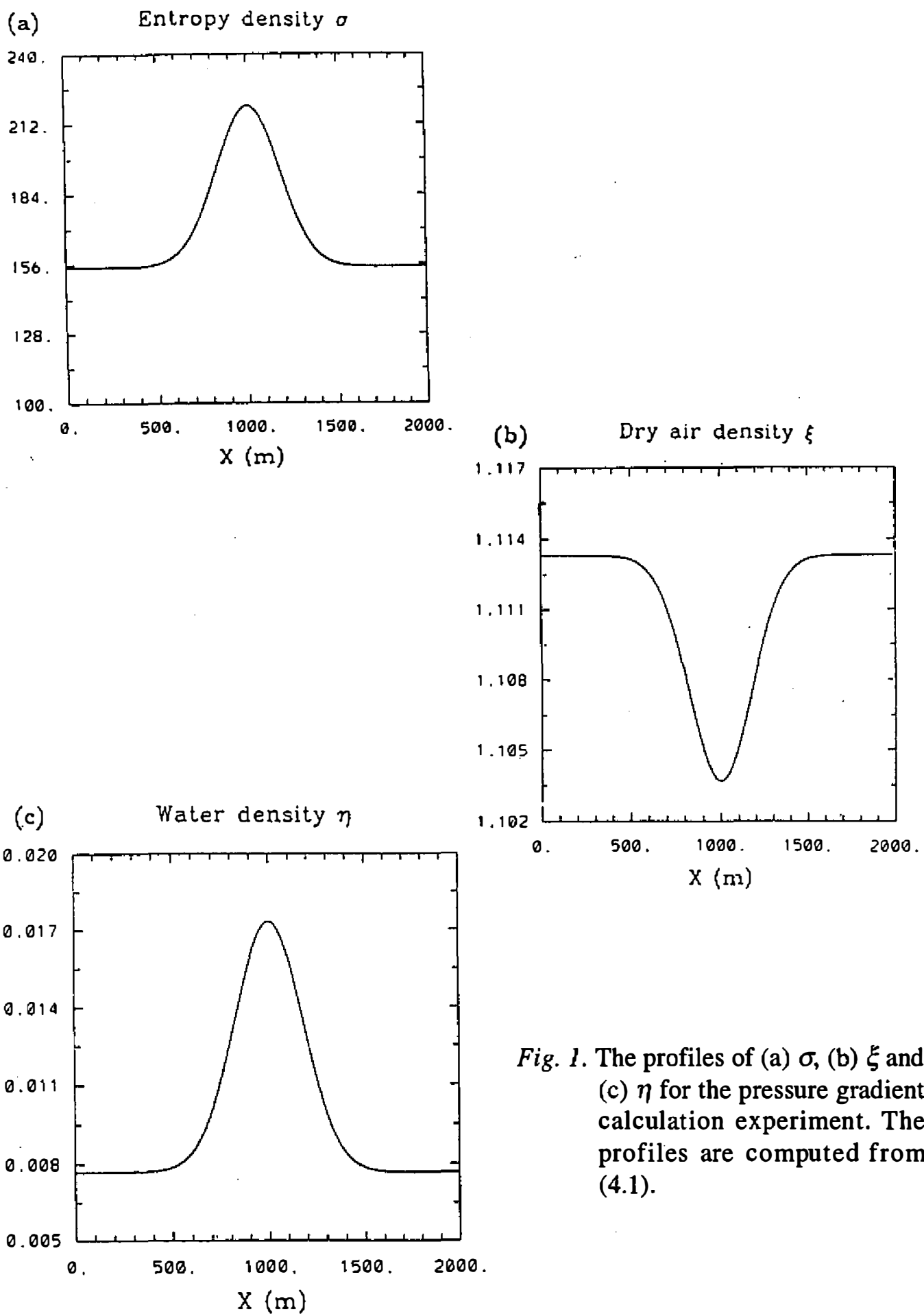

Fig. 1. The profiles of (a) $\sigma$, (b) $\xi$ and (c) $\eta$ for the pressure gradient calculation experiment. The profiles are computed from (4.1). 


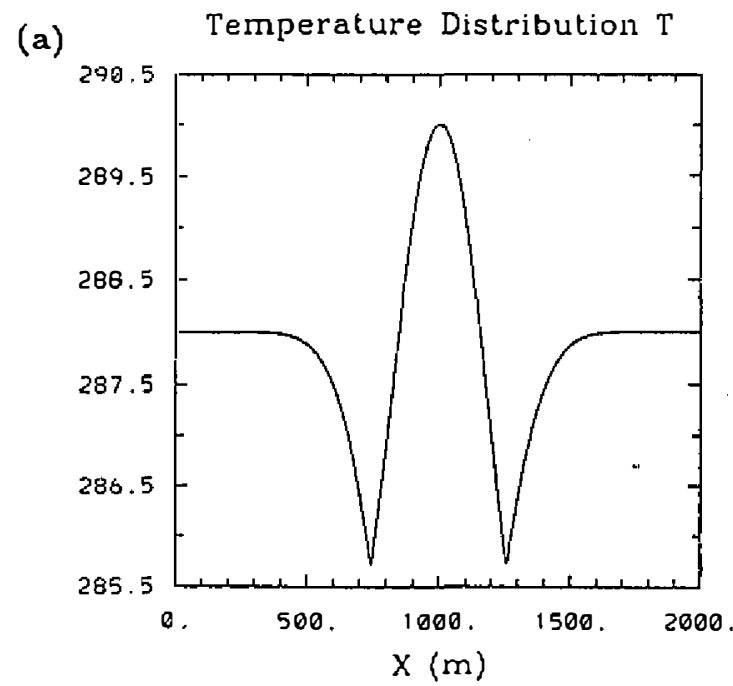

(b) Pressure Distribution $P$

(c) Liquid Water density
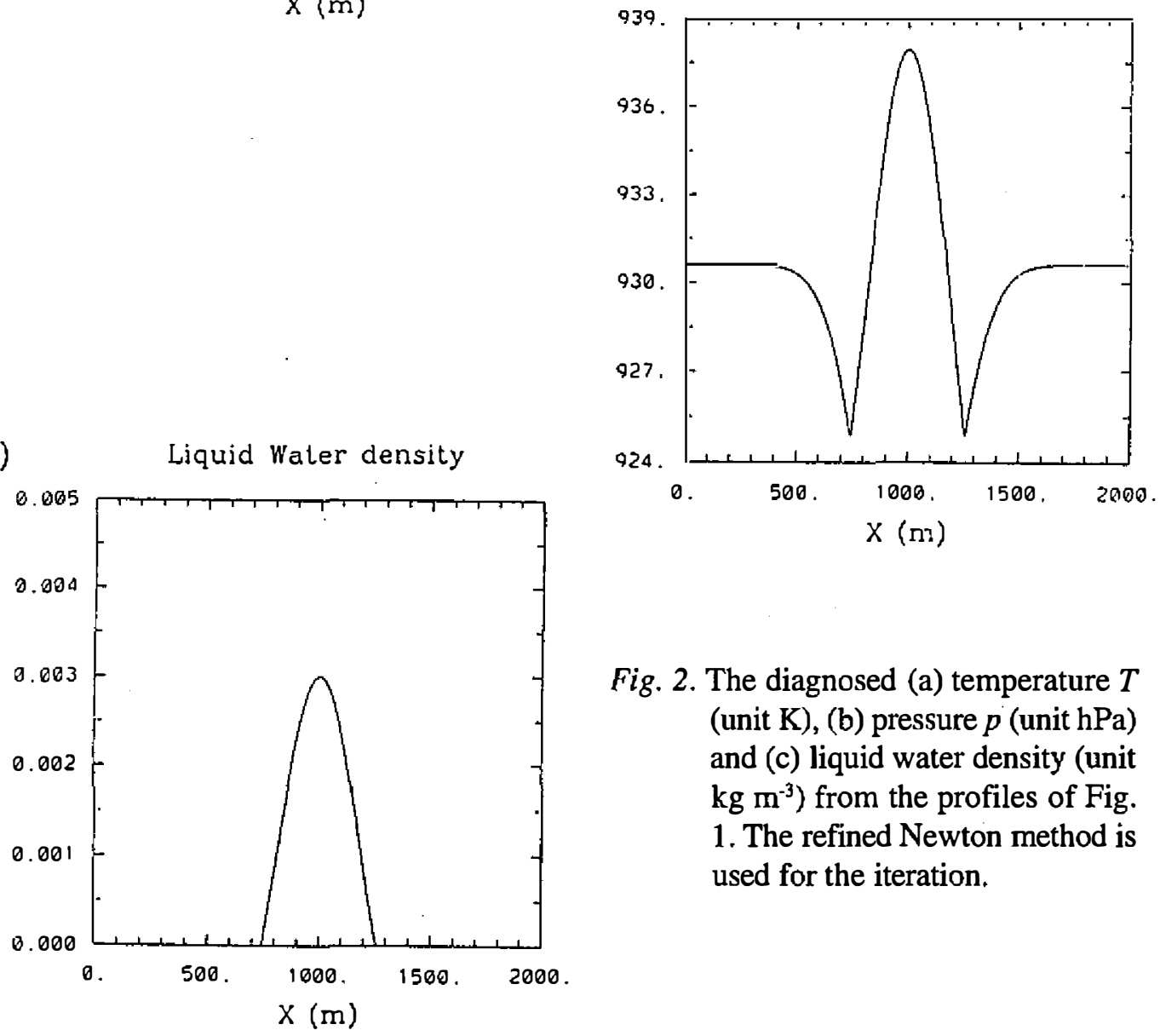

Fig. 2. The diagnosed (a) temperature $T$ (unit $\mathrm{K}$ ), (b) pressure $p$ (unit $\mathrm{hPa}$ ) and (c) liquid water density (unit $\mathrm{kg} \mathrm{m}^{-3}$ ) from the profiles of Fig. 1. The refined Newton method is used for the iteration. 
Pressure Gradient $\nabla P$

(a)

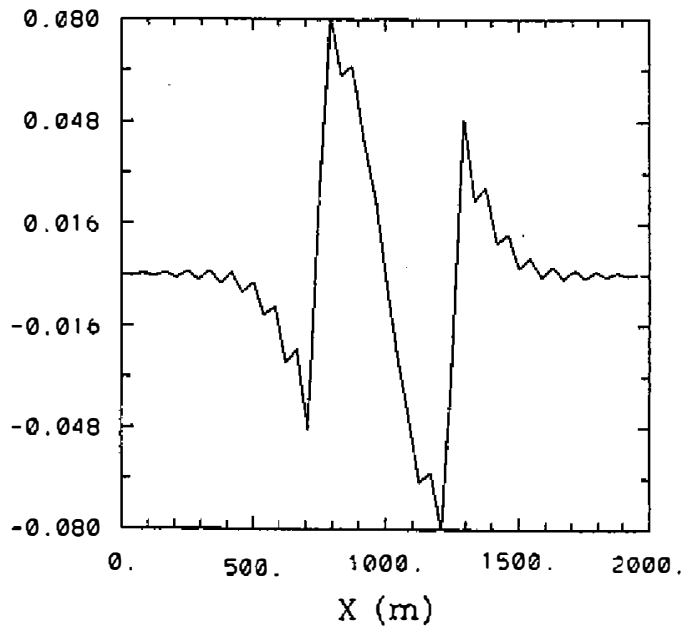

Pressure Gradient DP

(b)

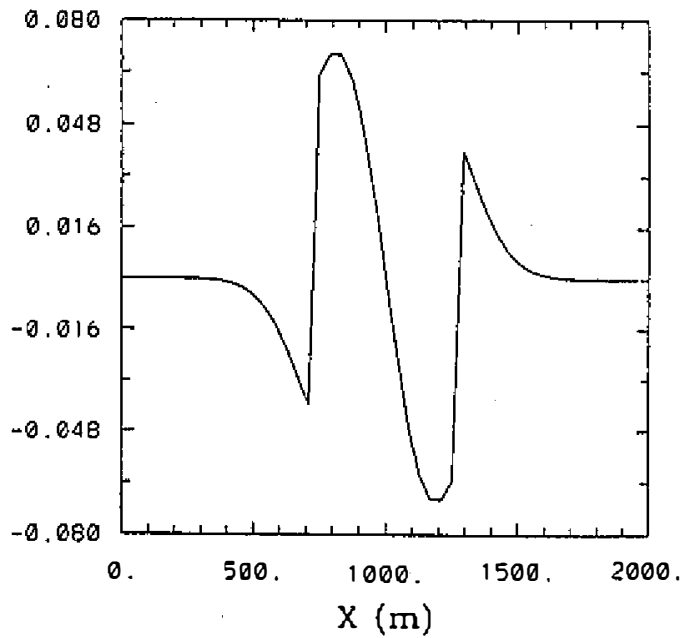

Fig. 3. The corresponding pressure gradient $\nabla_{p}$ of Fig. $2 b$ from (a) direct differentiation of the $p$ profile of Fig. $2 b$ by 48 points Fourier spectral method and from (b) Fourier specwal method with 48 points by (3.10) and (3.11).

of the spectral variables. To evaluate the quadratic term exactly, $48 \times 48$ collocation points are used. The Lanczos filter used is

$$
f_{m n}=\left(\frac{\sin (m \pi / M)}{m \pi / M}\right)\left(\frac{\sin (n \pi / N)}{n \pi / N}\right) .
$$

The second experiment (EXP2) is a dry hydrostatic adjustment experiment. We consider a basic state of 


$$
\begin{gathered}
\bar{T}(z)=293.15-\frac{g}{R_{d}} z,(K) \\
\bar{\xi}(z)=1.1478\left(\mathrm{kgm}^{-3}\right), \\
\bar{\eta}=0\left(\mathrm{kgm}^{-3}\right),
\end{gathered}
$$

The basic state satisfies hydrostatic balance. Superimposed on the basic state is a temperature anomaly defined by

$$
T^{\prime}=\Delta T \exp \left[-\left(\frac{x-1250}{200}\right)^{2}\right] \exp \left[-\left(\frac{z-1250}{200}\right)^{2}\right] .
$$

We have also set the $\xi^{\prime}$ equal to zero in our initial condition. Thus only the $p^{\prime}$ and $\sigma^{\prime}$ anomaly exist along with the $T^{\prime}$ anomaly. Since $\xi^{\prime}=0$, the anomaly (bubble) has no buoyancy and will not rise. Moreover, hydrostatic balance is violated because we have a $p^{\prime}$ but not a $\xi^{\prime}$ superimposed on the hydrostatic basic state.

Figure 4 shows the $U, W, \sigma^{\prime}, \xi^{\prime}, p^{\prime}$ and $T^{\prime \prime}$ in physical domain at time $0.3 \mathrm{~s}$ for the calculation with $\Delta T=2.5 \mathrm{~K}$ in (4.3). The perturbation temperature in Fig. $4 \mathrm{~d}$ is given by $T^{\prime \prime}$ $=T-\bar{T}-T^{\prime}$, the difference between $T$ and the initial $T$. Figures 5 and 6 are similar to Fig. 4 except at time $1.8 \mathrm{~s}$ and $30 \mathrm{~s}$ respectively. Figure 6 reveals the motion and density fields associated with a rising bubble. This rising bubble (now $\xi^{\prime}<0$ ) can be viewed as the result of the hydrostatic adjustment by the acoustic waves. In contrast, Figs. 4 and and 5 indicate the motion and density fields associated with the transient acoustic waves.

To see how fast the acoustic wave can make the hydrostatic adjustment, we have plotted the time series at the center of the domain for the variables of divergence, $T^{\prime}, \xi^{\prime}$ and $p^{\prime}$ in Fig. 7. Figure 7 indicates that it takes about 3 to 4 seconds for these variables to reach a steady state. Figure 8 is similar to Fig. 7 except for an experiment with $\Delta T=7.5 \mathrm{~K}$ in (4.3). Interestingly, the time series in both cases are very similar. This indicates the atmosphere reaches the "anelastic balance" $\left(\partial p^{\prime} / \partial t \approx 0\right.$ and $\left.\partial U / \partial x+\partial W / \partial z \approx 0\right)$ or converts a zero $\xi^{\prime}$ to a finite value of $\xi^{\prime}$ in 3 to 4 seconds, regardless of the size of $\Delta T$. In other words, anelastic models are just as good as compressible models if the transient acoustic waves are not the focus of modeling. Discussions on one-dimensional acoustic adjustment with an isothermal basic state can be found in Bannon (1995). Duffy (1997) examined hydrostatic adjustment through the generation of acoustic-gravity waves.

The third experiment (EXP3) is a rising dry bubble in a hydrostatic atmosphere. We consider a basic state

$$
\bar{T}(z)=293.15-\frac{g}{C_{p d}} z,(K)
$$




$$
U, W \& \sigma^{\prime} \quad 0.30 \mathrm{sec}
$$

(a)

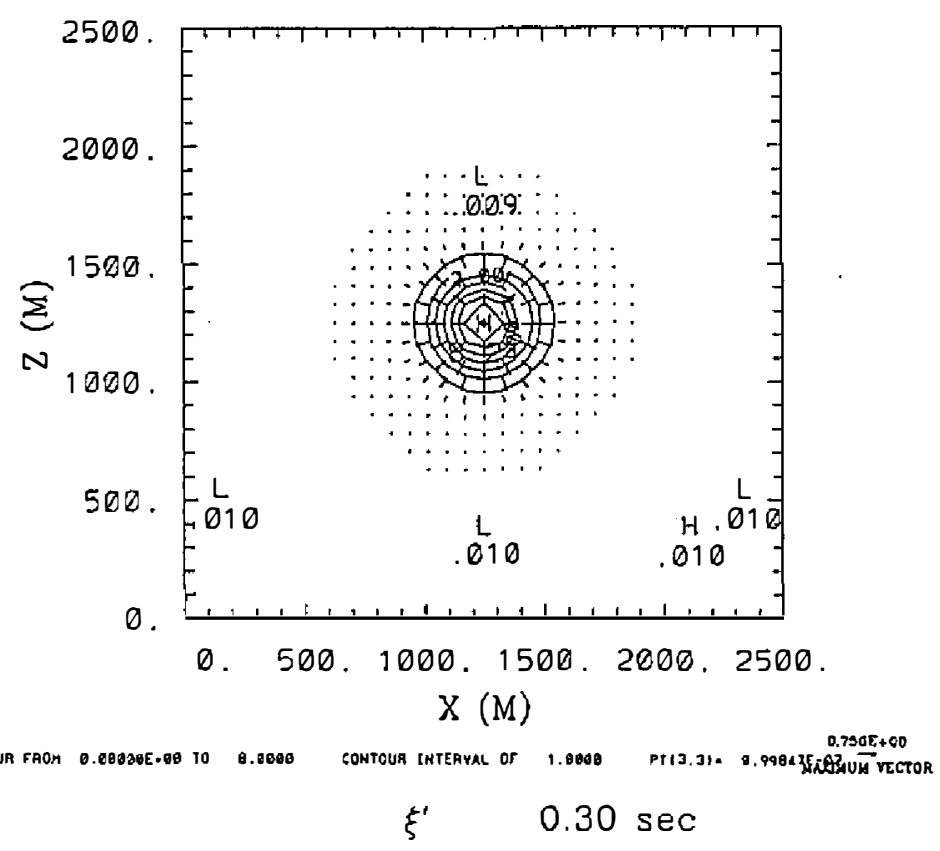

(b)

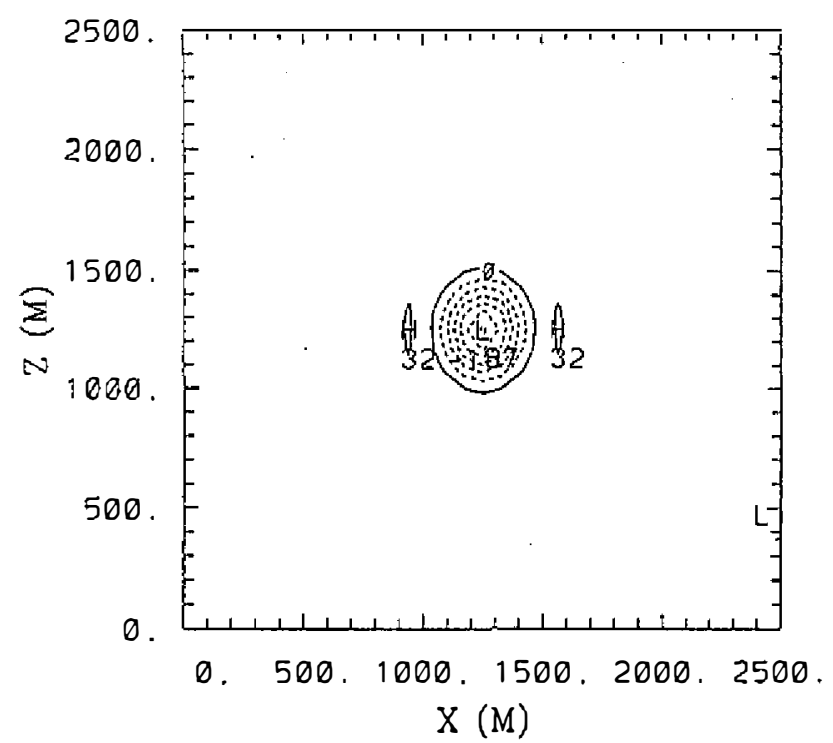

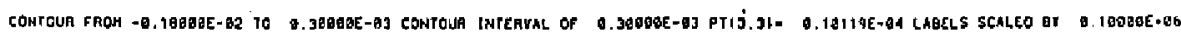

Fig. 4. The numerical results of EXP2 in $x-z$ domain at $t=0.3 \mathrm{~s}$ for (a) the $U, W$ (the maximum vector is $0.756 \mathrm{~kg} \mathrm{~m}^{-2} \mathrm{~s}^{-1}$ ) and $\sigma^{\prime}$ (unit $\mathrm{J} \mathrm{m}^{-3} \mathrm{~K}^{-1}$, contour interval $1 \mathrm{~J} \mathrm{~m}^{-3} \mathrm{~K}^{-1}$ ), (b) $\xi^{\prime}$, (unit $10^{-5} \mathrm{~kg} \mathrm{~m}^{-3}$, contour interval $3 \times 10^{-4} \mathrm{~kg}$ $\mathrm{m}^{-3}$ ), (c) $p^{\prime}$ (unit $\mathrm{hPa}$, contour interval $0.9 \mathrm{hPa}$ ) and (d) $T^{\prime \prime}$, the difference between $T$ and the initial $T$, (unit $10^{-3} \mathrm{~K}$, contour interval $0.02 \mathrm{~K}$ ). 


$$
\mathrm{P}^{\prime} \quad 0.30 \mathrm{sec}^{\prime}
$$

(c)

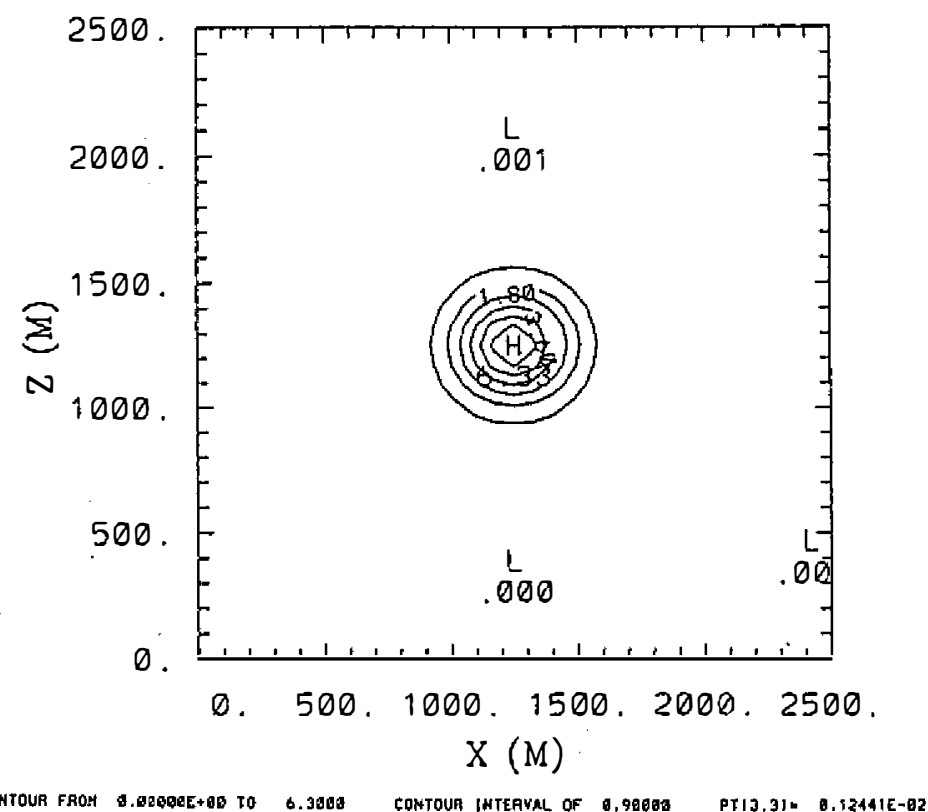

(d)

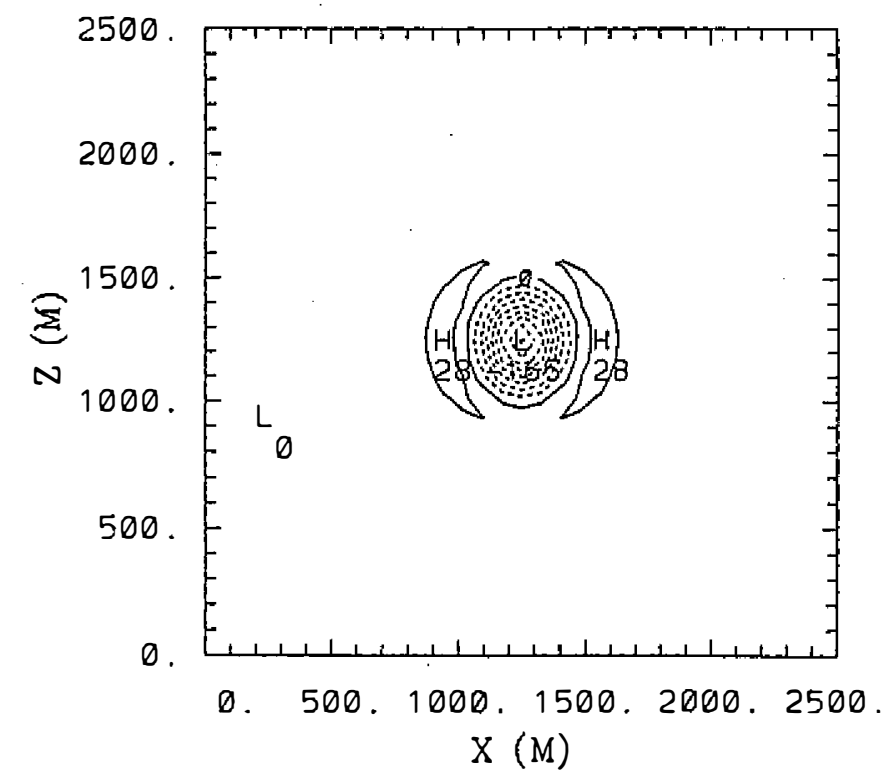


(a)

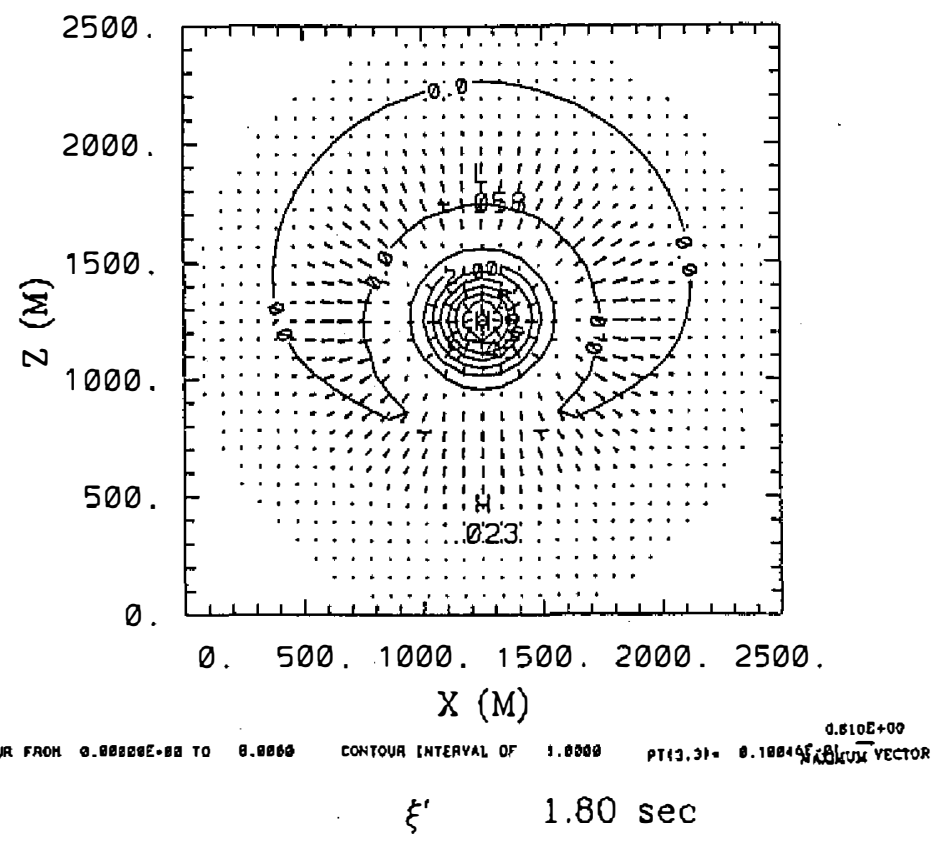

(b)

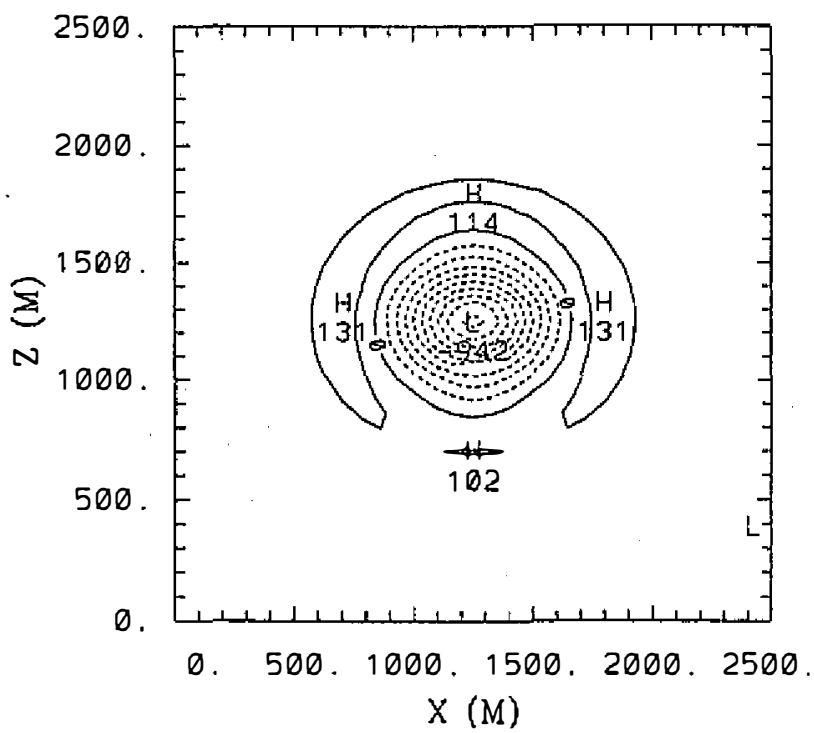

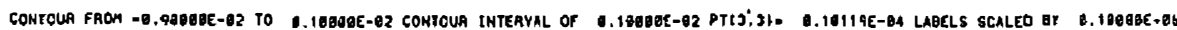

Fig. 5. Same as Fig. 4 except at $t=1.8 \mathrm{~s}$ with (a) the $\mathrm{U}, W$ (the maximum vector is $0.61 \mathrm{~kg} \mathrm{~m}^{-2} \mathrm{~s}^{-1}$ ) and $\sigma^{\prime}$ (unit $\mathrm{J} \mathrm{m}^{-3} \mathrm{~K}^{-1}$, contour interval $1 \mathrm{~J} \mathrm{~m}^{-3} \mathrm{~K}^{-1}$ ), (b) $\xi^{\prime}$, (unit $10^{-5} \mathrm{~kg} \mathrm{~m}^{-3}$, contour interval $1 \times 10^{-3} \mathrm{~kg} \mathrm{~m}^{-3}$ ), (c) $p^{\prime}$ (unit $\mathrm{hPa}$, contour interval $0.5 \mathrm{hPa}$ ) and (d) $T^{\prime \prime}$, the difference between $T$ and the initial $T$, (unit $\mathrm{K}$, contour interval $0.1 \mathrm{~K}$ ). 


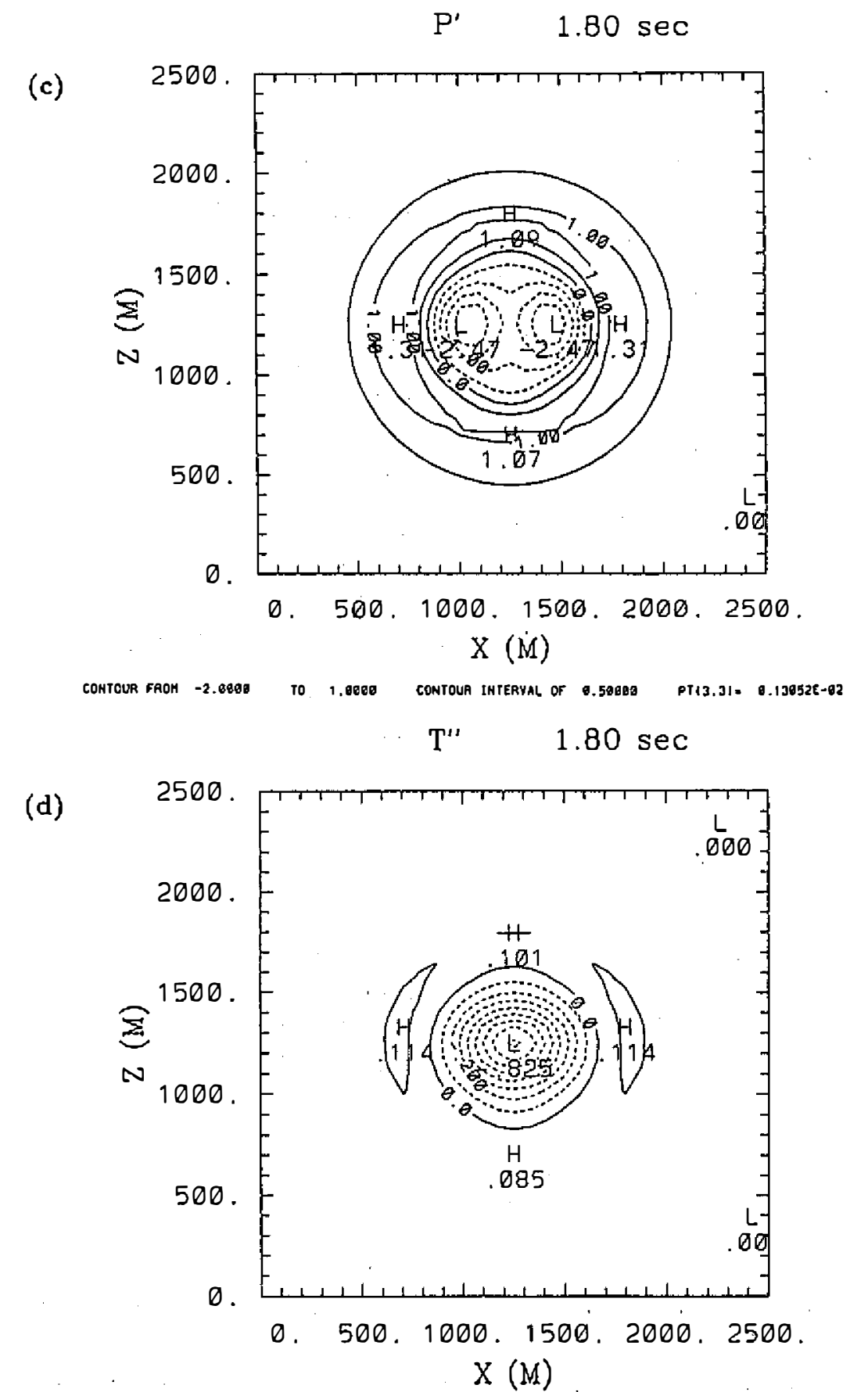

CONTOUA FAOH -6.80000

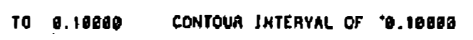

PTiJ.31" (0.16184E-03

(Fig. 5. continued) 


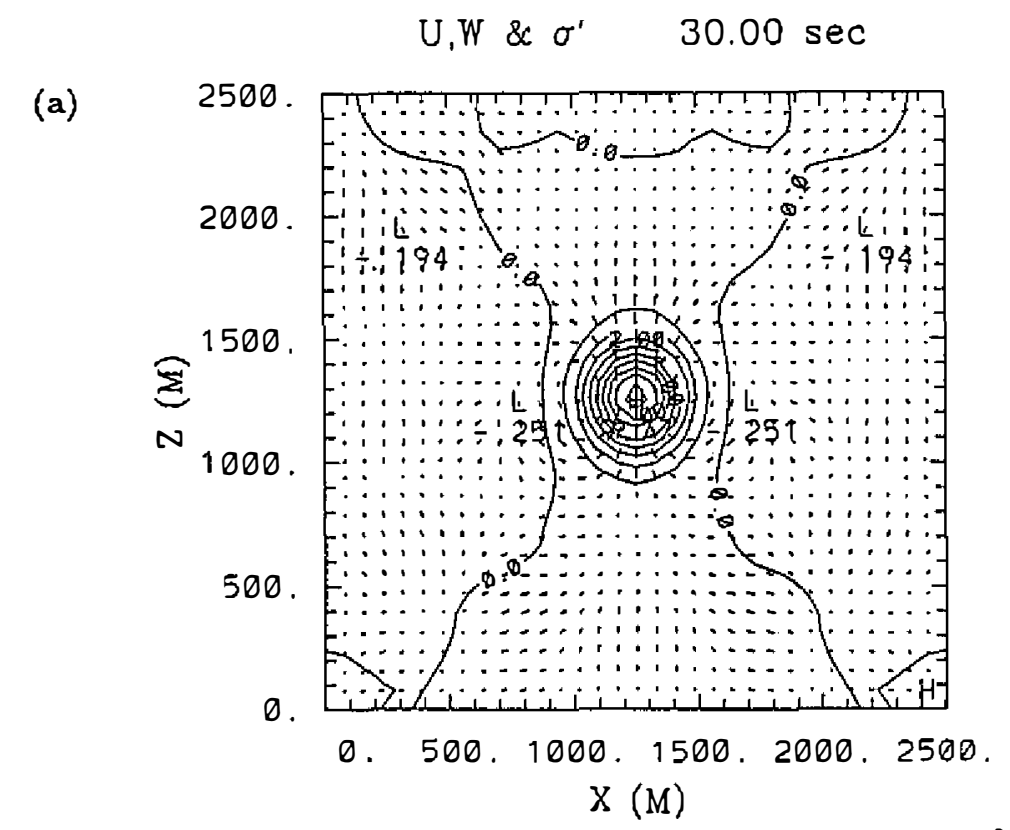

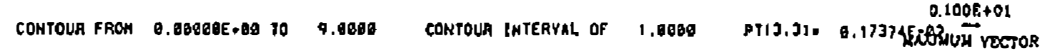

(b)

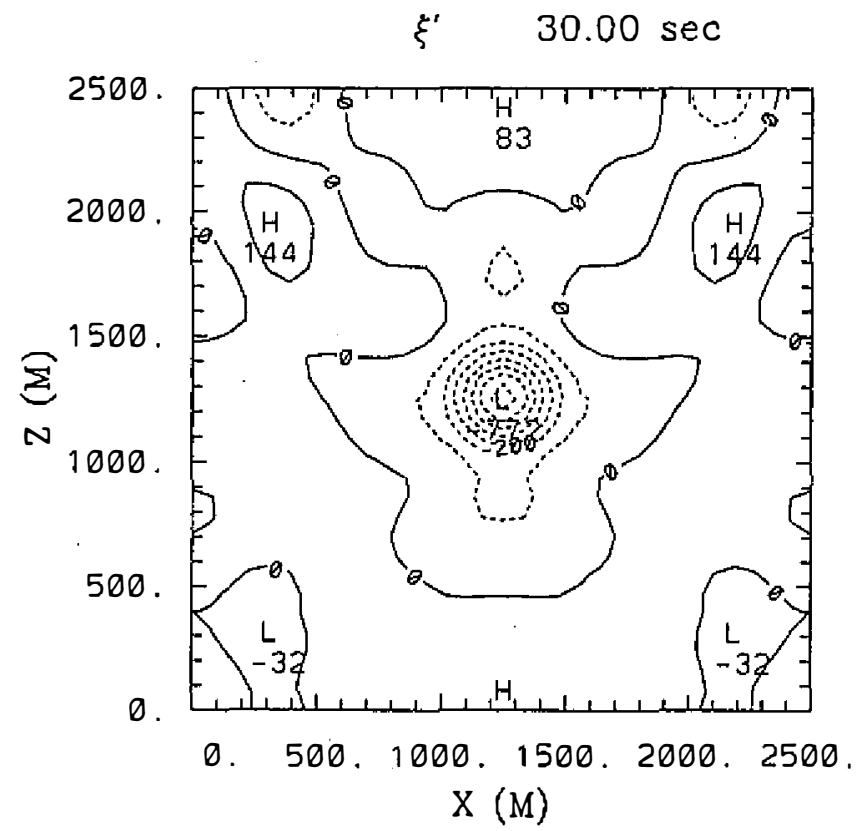

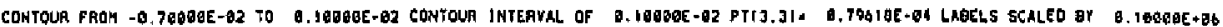

Fig. 6. Same as Fig. 4 except at $t=30.0$ s with (a) the $U, W$ (the maximum vector is $1 \mathrm{~kg} \mathrm{~m}^{-2} \mathrm{~s}^{-1}$ ) and $\sigma^{\prime}$ (unit $\mathrm{J} \mathrm{m}^{-3} \mathrm{~K}^{-1}$, contour interval $1 \mathrm{~J} \mathrm{~m}^{-3} \mathrm{~K}^{-1}$ ) and (b) $\xi^{\prime}$, (unit $10^{-5} \mathrm{~kg} \mathrm{~m}^{-3}$, contour interval $1 \times 10^{-3} \mathrm{~kg} \mathrm{~m}^{-3}$ ). 
Time Series of Divergence

(a)

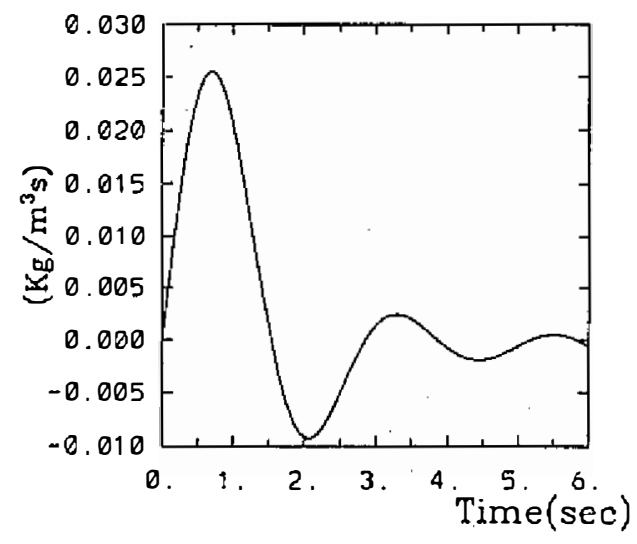

Time Series of $\mathrm{T}^{\prime}$

(b)

(K)

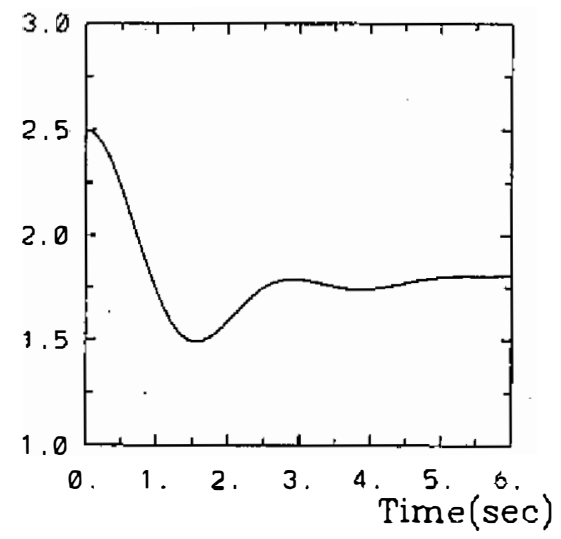

(c)

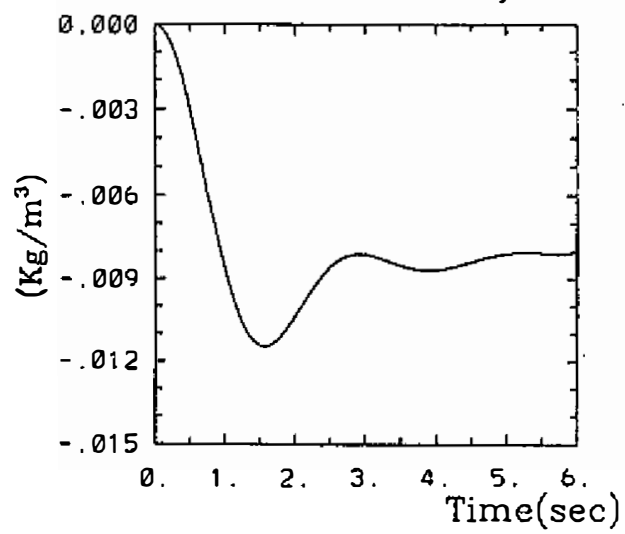

Time Series of $\mathrm{P}^{\prime}$

(d)

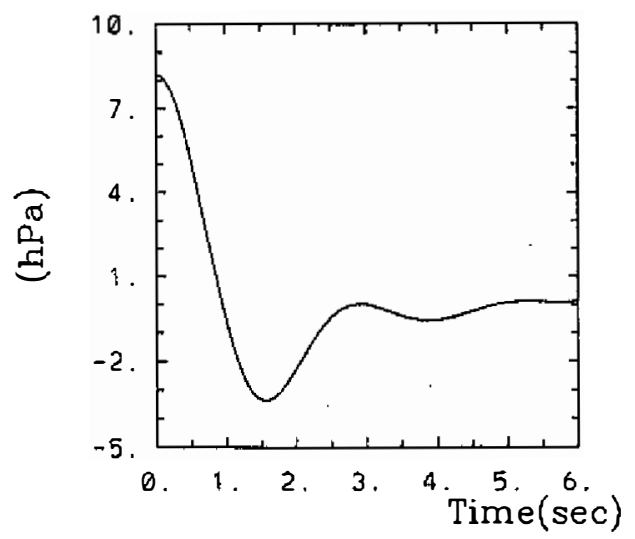

Fig. 7. The time series of EXP2 for (a) momentum density divergence (unit kg $\mathrm{m}^{-3} \mathrm{~s}^{-1}$ ), (b) $T^{\prime}$ (unit $\mathrm{K}$ ), (c) $\xi^{\prime}$ (unit $\mathrm{kg} \mathrm{m}^{-3}$ ) and (d) $p^{\prime}$ (unit $\mathrm{hPa}$ ) at the center of domain with a $\Delta T=2.5 \mathrm{~K}$ in (4.3).

$$
\begin{gathered}
\bar{\xi}(z)=1.275\left(\frac{\bar{T}(z)}{293.15}\right)^{\frac{c_{w d}}{R_{d}}},\left(\mathrm{kgm}^{-3}\right) \\
\bar{\eta}=0 .\left(\mathrm{kgm}^{-3}\right)
\end{gathered}
$$

Superimposed on (4.4) is a temperature anomaly given by

$$
T^{\prime}(x, z)=2.5 \exp \left[-\left(\frac{x-1250}{200}\right)^{2}\right] \exp \left[-\left(\frac{x-625}{200}\right)^{2}\right]
$$


Time Series of Divergence

(a)

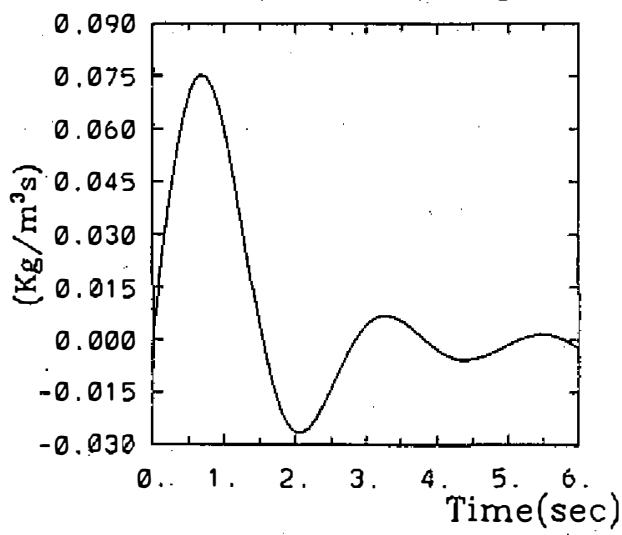

Time Series of $T^{\prime}$

(b)

(K)

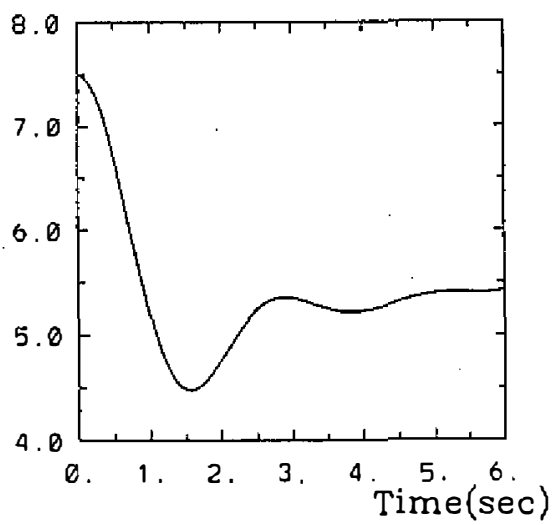

(c)

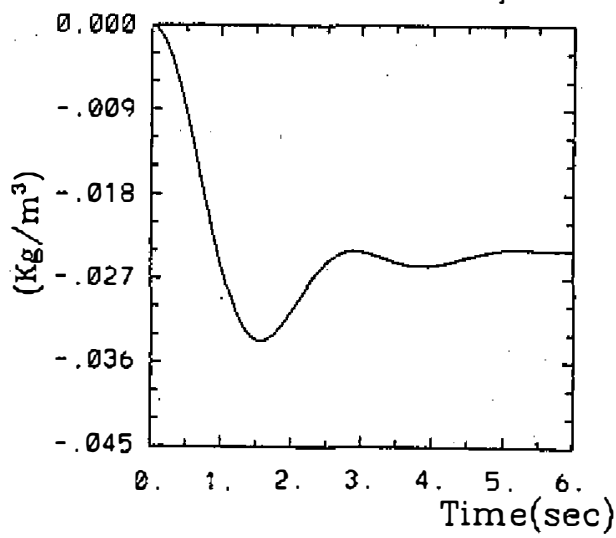

(d)

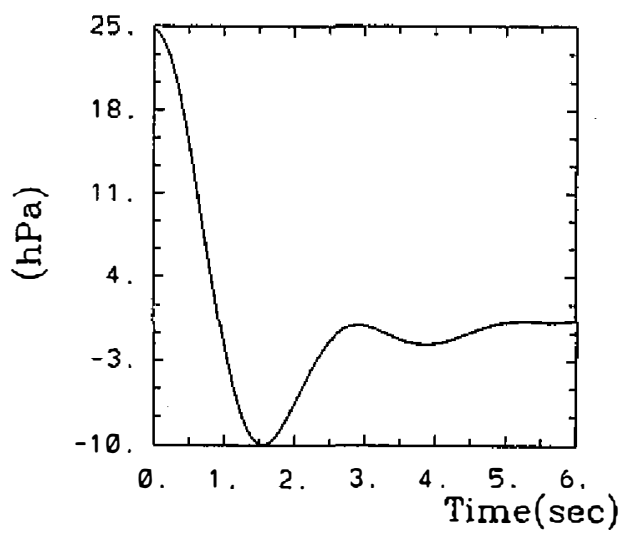

Fig. 8. Same as Fig. 7 except with a $\Delta \mathrm{T}=7.5 \mathrm{~K}$ in (4.3).

Namely, we have the initial condition for our third experiment

$$
\begin{gathered}
T(x, y)=\bar{T}(z)+T^{\prime}(x, z), \\
\xi^{\prime}(x, z)=\bar{\xi}(z)+\frac{\bar{T}(z)}{T(x, z)}, \\
\eta^{\prime}(x, z)=0 .
\end{gathered}
$$

The experiment is designed so that $p^{\prime} \approx 0$ in the initial condition. Thus, contrary to the second experiment, we do not experience significant acoustic wave radiation in this experi- 
ment. Figures 9 and 10 are the $U, W, \sigma^{\prime}, T^{\prime}, p^{\prime}$ and $\xi^{\prime}$ in physical space for $t=150 \mathrm{~s}$ and $t=300$ s respectively. We observe strong updraft in the center of the warm bubble and relatively weak downward motion in a broad area adjacent to the rising bubble. On top of the rising bubble there is high pressure while below the bubble top is a slightly broad area of low pressure.

Figure 11 shows the time series in the first stage of EXP3 $(t<7.5 \mathrm{~s})$ at the point $(x=$ $1250 \mathrm{~m}, z=780 \mathrm{~m}$ ) for divergence of momentum density, the difference between $T$ and the initial $T$ (i.e., $T^{\prime \prime}$ ), the difference between $\xi$ and the initial $\xi$ (i.e., $\xi^{\prime \prime}$ ), and the perturbation pressure $p^{\prime}$. The point $(x=1250 \mathrm{~m}, z=780 \mathrm{~m})$ is above the rising warm bubble where we expect a region of high pressure. Figure 11 indicates the presence of acoustic waves in that $\xi^{\prime \prime}$ is out of phase with the momentum density divergence. The formation of a high pressure region above a warm rising bubble is associated with a series of transient acoustic waves.

The fourth experiment (EXP4) is a rising moist bubble experiment in a hydrostatic atmosphere. We consider a basic state of

$$
\begin{gathered}
\bar{T}(z)=293.15-\frac{g}{C_{p d}} z,(K) \\
\bar{r}_{t}=0.0135 \exp \left(-\frac{z}{1500}\right), \\
\bar{\xi}(z)=1.275\left(\frac{\bar{T}}{239.15}\right)^{\frac{c_{v d}}{R_{d}}} \exp \left[\frac{R_{v}}{R_{d}}\left(0.0135-\bar{r}_{t}\right)\right],\left(\mathrm{kgm}^{-3}\right) \\
\bar{\eta}=\bar{r}_{t} \bar{\xi},\left(\mathrm{kgm}^{-3}\right)
\end{gathered}
$$

where $\bar{r}_{t}$ is the mixing ratio of total water density with respect to the dry air density $\xi$. The basic state dry air density in (4.7c) is computed from the equation of state. Now we consider a moist bubble with temperature perturbation $T^{\prime}$ given by (4.5)

$$
\begin{aligned}
& T(x, z)=\bar{T}(z)+T^{\prime}(x, z), \\
& \xi^{\prime}(x, z)=\bar{\xi} \frac{\bar{T}}{T(x, z)}-\bar{\xi}, \\
& \eta^{\prime}(x, z)=\left(\bar{\xi}+\xi^{\prime}(x, z)\right) \bar{r}_{t} .
\end{aligned}
$$


U,W \& $\sigma^{\prime} \quad 150.00 \mathrm{sec}$

(a)

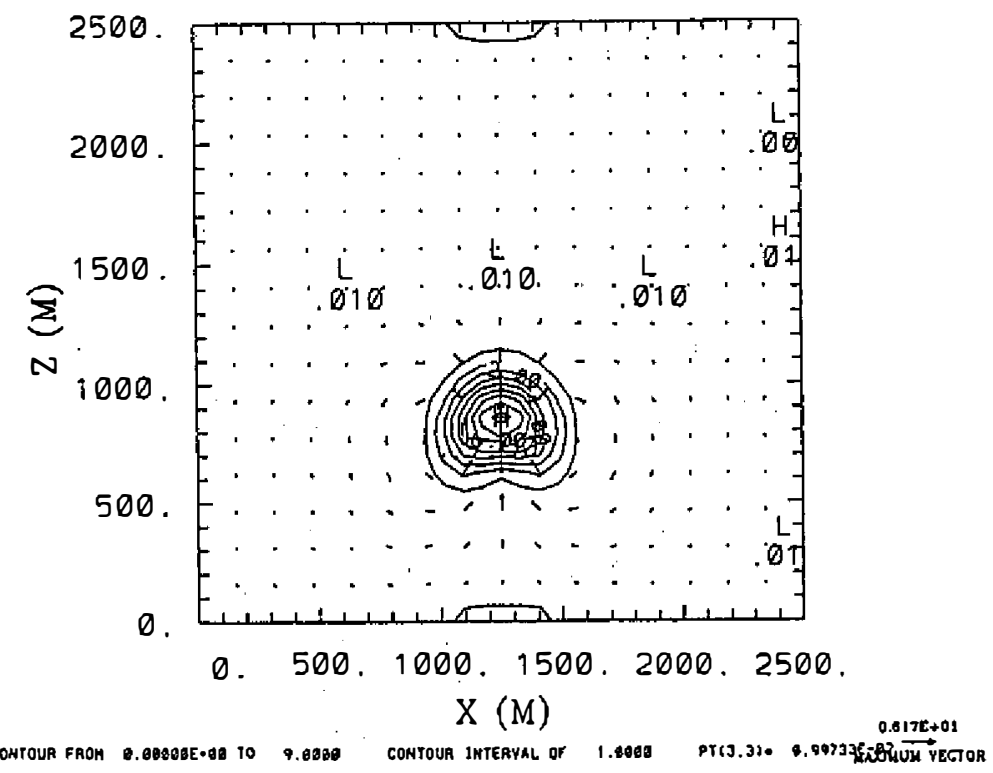

(b)

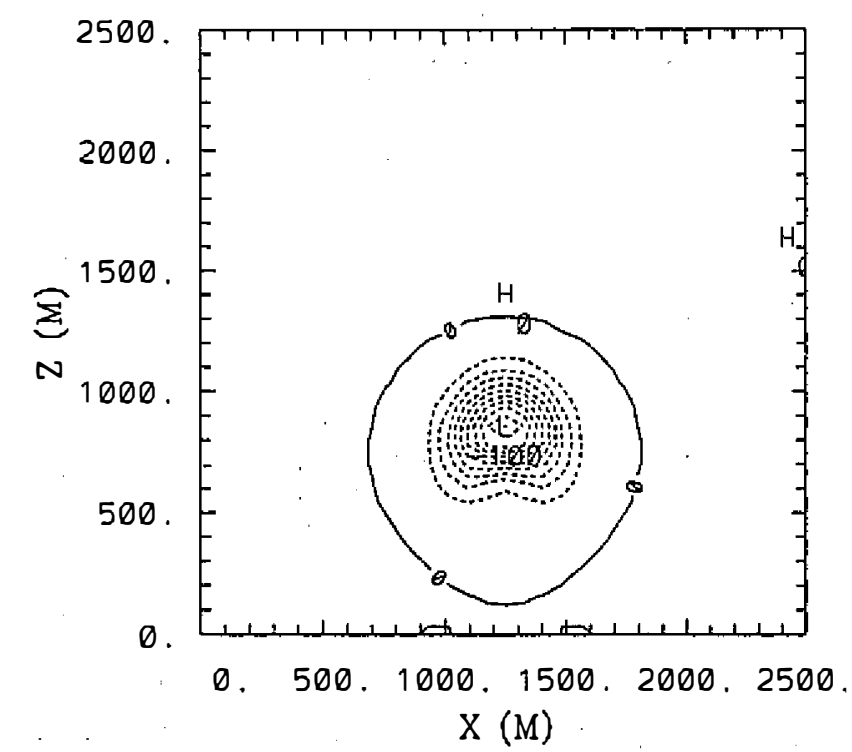

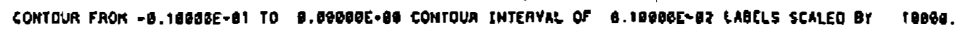

Fig. 9. The numerical results of EXP3 in $x$-z domain at $t=150$ s for (a) the $U, W$ (the maximum vector is $6.17 \mathrm{~kg} \mathrm{~m}^{-2} \mathrm{~s}^{-1}$ ) and $\sigma^{\prime}$ (unit $\mathrm{J} \mathrm{m}^{-3} \mathrm{~K}^{-1}$, contour interval $1 \mathrm{~J} \mathrm{~m}^{-3} \mathrm{~K}^{-1}$ ), (b) $\xi^{\prime}$, (unit $10^{-4} \mathrm{~kg} \mathrm{~m}^{-3}$, contour interval $10^{-3} \mathrm{~kg} \mathrm{~m}^{-3}$ ), (c) $p^{\prime}$ (unit $10^{-3} \mathrm{hPa}$, contour interval $0.02 \mathrm{hPa}$ ) and (d) $T^{\prime}$ (unit $\mathrm{K}$, contour interval $0.3 \mathrm{~K}$ ). 
(c)

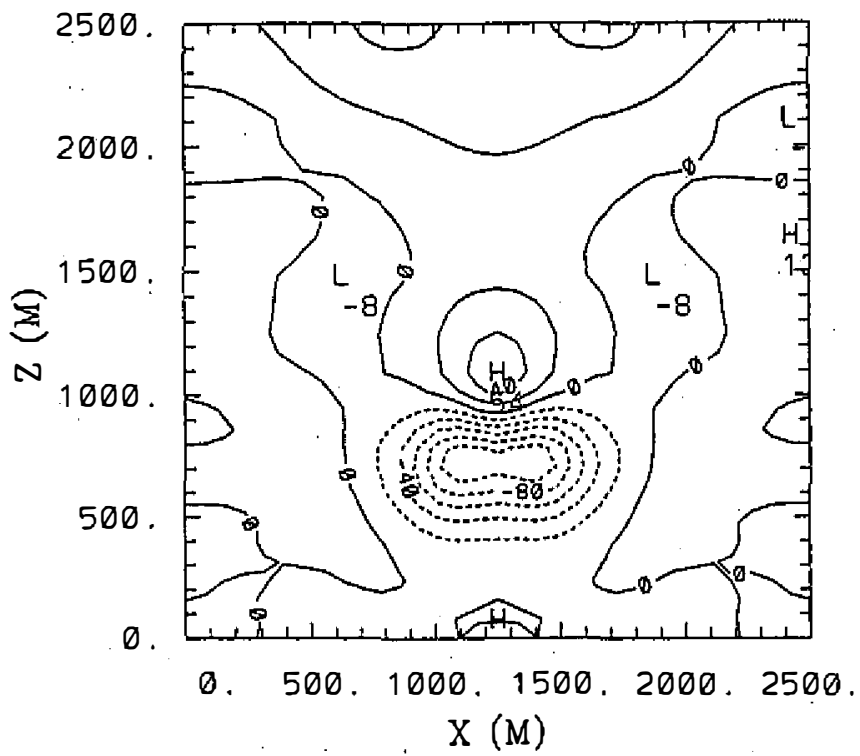

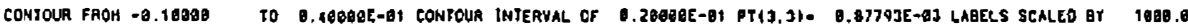

(d)

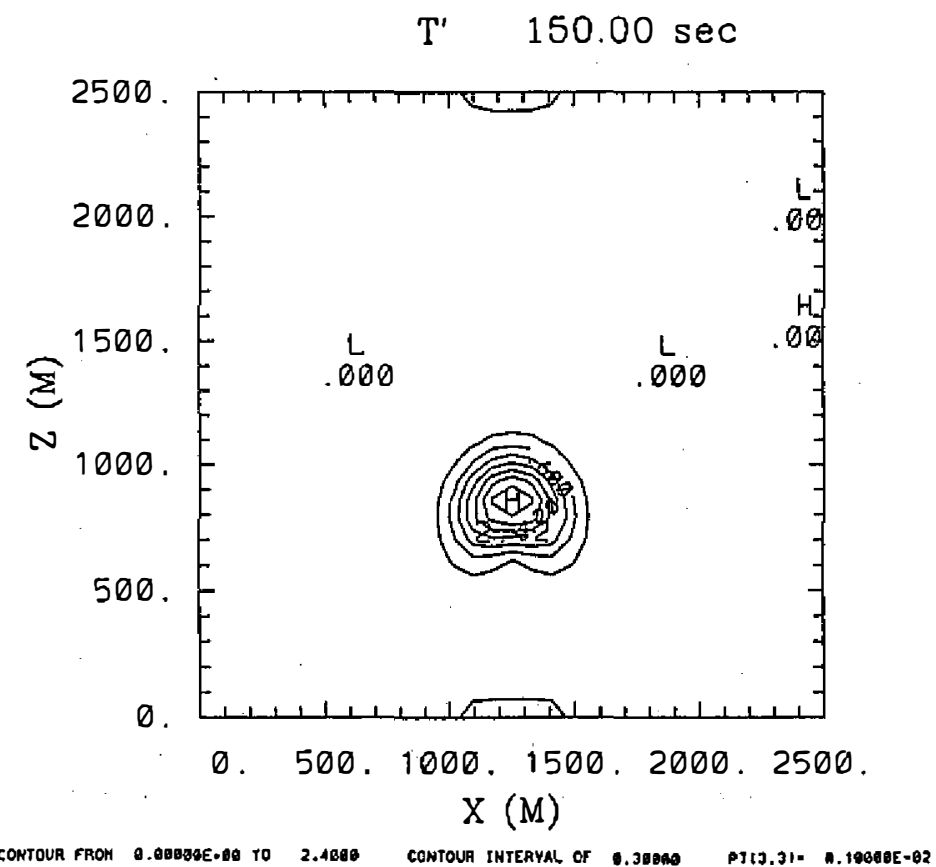

(Fig. 9. continued) 


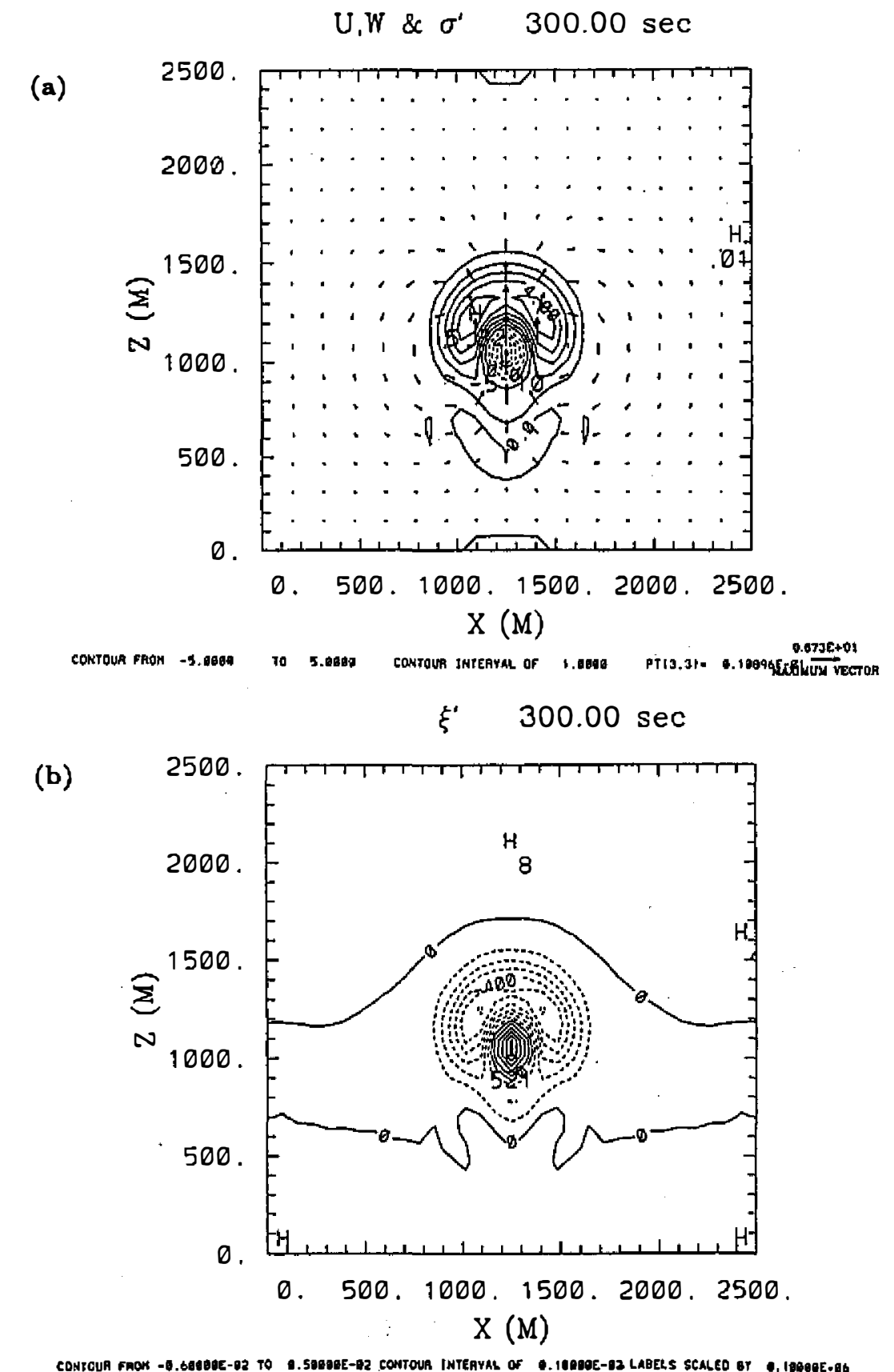

Fig. 10. Same as Fig. 9 except at $t=300$ s with (a) the $U, W$ (the maximum vector is $6.73 \mathrm{~kg} \mathrm{~m}^{-2} \mathrm{~s}^{-1}$ ) and $\sigma^{\prime}$ (unit $\mathrm{J} \mathrm{m}^{-3} \mathrm{~K}^{-1}$, contour interval $1 \mathrm{~J} \mathrm{~m}^{-3} \mathrm{~K}^{-1}$ ), (b) $\xi^{\prime}$, (unit $10^{-5} \mathrm{~kg} \mathrm{~m}^{-3}$, contour interval $10^{-3} \mathrm{~kg} \mathrm{~m}^{-3}$ ), (c) $p^{\prime}$ (unit $10^{-3} \mathrm{hPa}$, contour interval $0.04 \mathrm{hPa}$ ) and (d) $T^{\prime}$ (unit $\mathrm{K}$, contour interval $0.3 \mathrm{~K}$ ). 


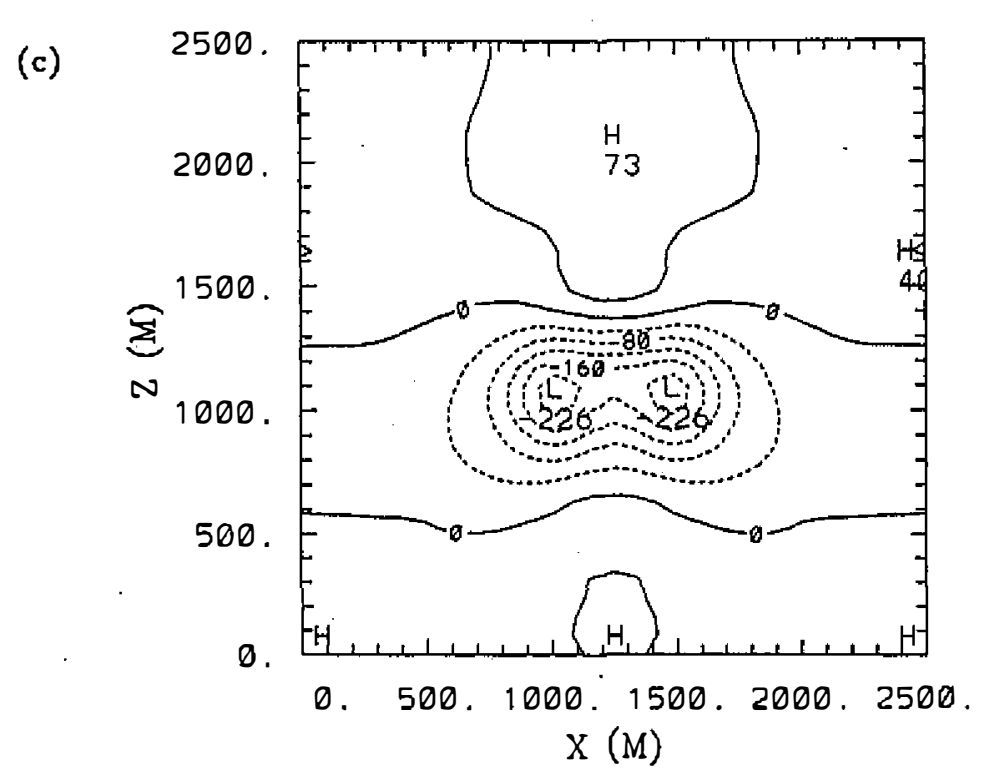

\section{$\mathrm{T}^{\prime} \quad 300.00 \mathrm{sec}$}

(d)

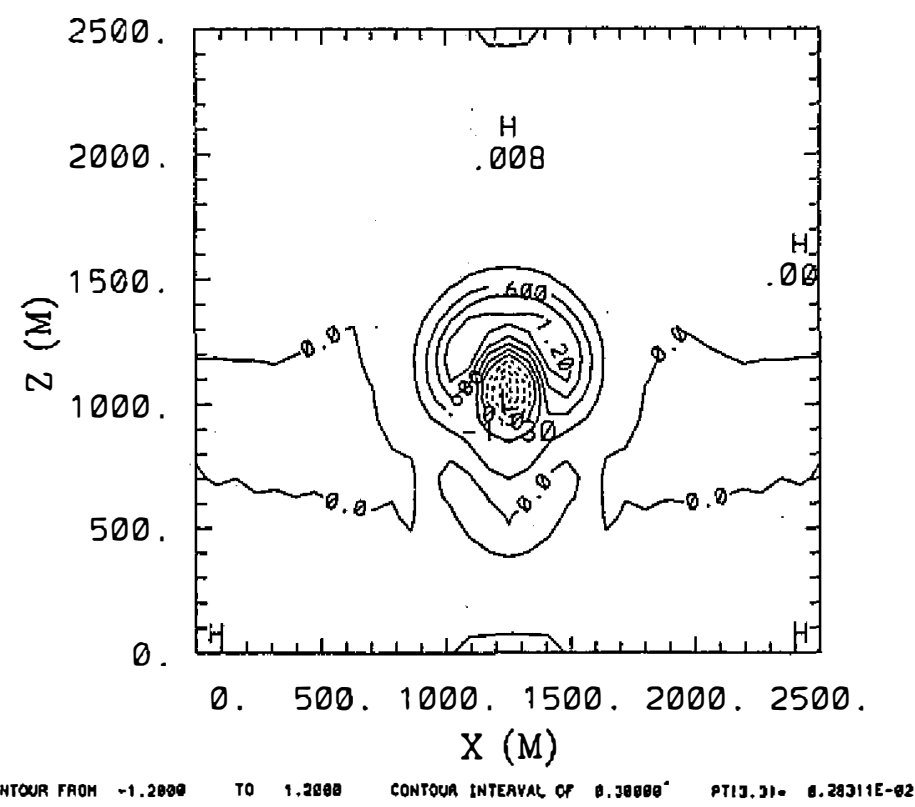

(Fig. 10. continued) 
Time Series of Divergence

(a)

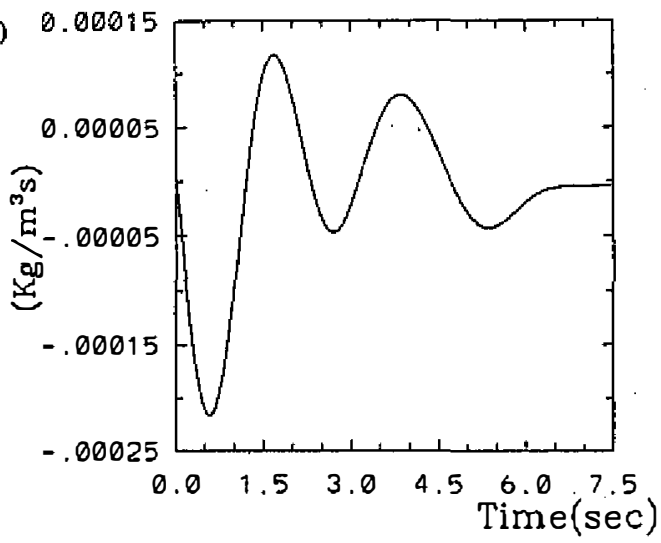

Time Series of $\Upsilon^{\prime \prime}$

(b)

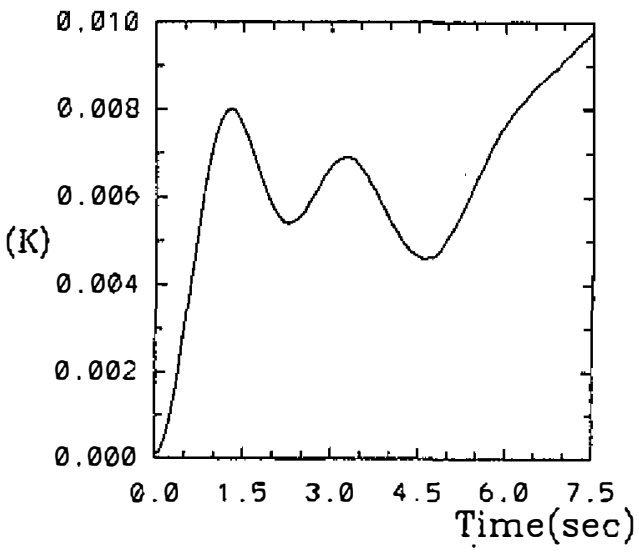

Time Series of $\xi^{\prime \prime}$

(c)

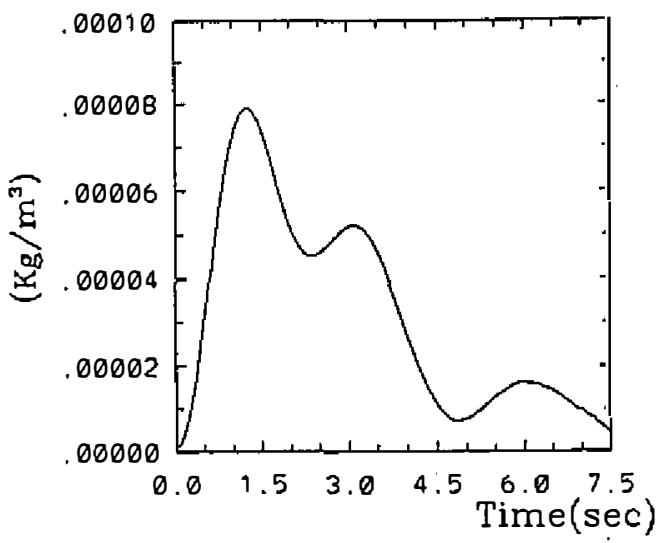

Time Series of $P^{\prime}$

(d)

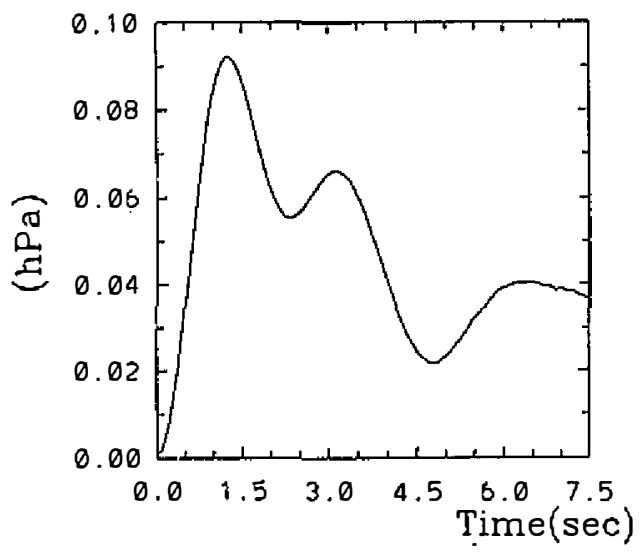

Fig. 11. Time series in the first stage of EXP3 $(t<7.5 \mathrm{~s})$ at the point $(x=1250 \mathrm{~m}$, $z=780 \mathrm{~m}$ ) for (a) divergence of momentum density (unit $\mathrm{kg} \mathrm{m}^{-3} \mathrm{~s}^{-1}$ ) (b) $T^{\prime \prime}$, the difference between $T$ and the initial $T$ (unit $\mathrm{K}$ ), (c) $\xi^{\prime \prime}$, the difference between $\xi$ and the initial $\xi$ (unit $\mathrm{kg} \mathrm{m}^{-3}$ ) and (d) perturbation pressure $p^{\prime}$ (unit $\mathrm{hPa}$ ).

Equation (4.8) gives positive values of $\sigma^{\prime}, T^{\prime}$ and $\eta^{\prime}$ and a negative value of $\xi^{\prime}$ (positive buoyancy) for the bubble. Figures 12 and 13 are the results at $t=150 \mathrm{~s}$ and $t=240 \mathrm{~s}$ respectively for EXP4. With the initial maximum $T$ of $2.5 \mathrm{~K}$, the results indicate that the perturbation temperature $T^{\prime}$ decreases with time before the condensation takes place (i.e., $t=150 \mathrm{~s}$ ). From Fig. 13 we see that the maximum perturbation temperature $T^{\prime}$ is about $4 \mathrm{~K}$ which is higher than the initial value of $2.5 \mathrm{~K}$. This is due to the release of the latent heat. Figure 13 also indicates a very small warming $(0.01 \mathrm{~K})$ and drying $\left(\eta^{\prime}=-0.0128 \mathrm{kgm}^{-3}\right)$ outside the cloud as a result of forced downward motion in a constant $\theta$ atmosphere. The perturbation pressure distribution is more complicated than the pressure distribution in Figs. 9 and 10. This is probably also due to the 


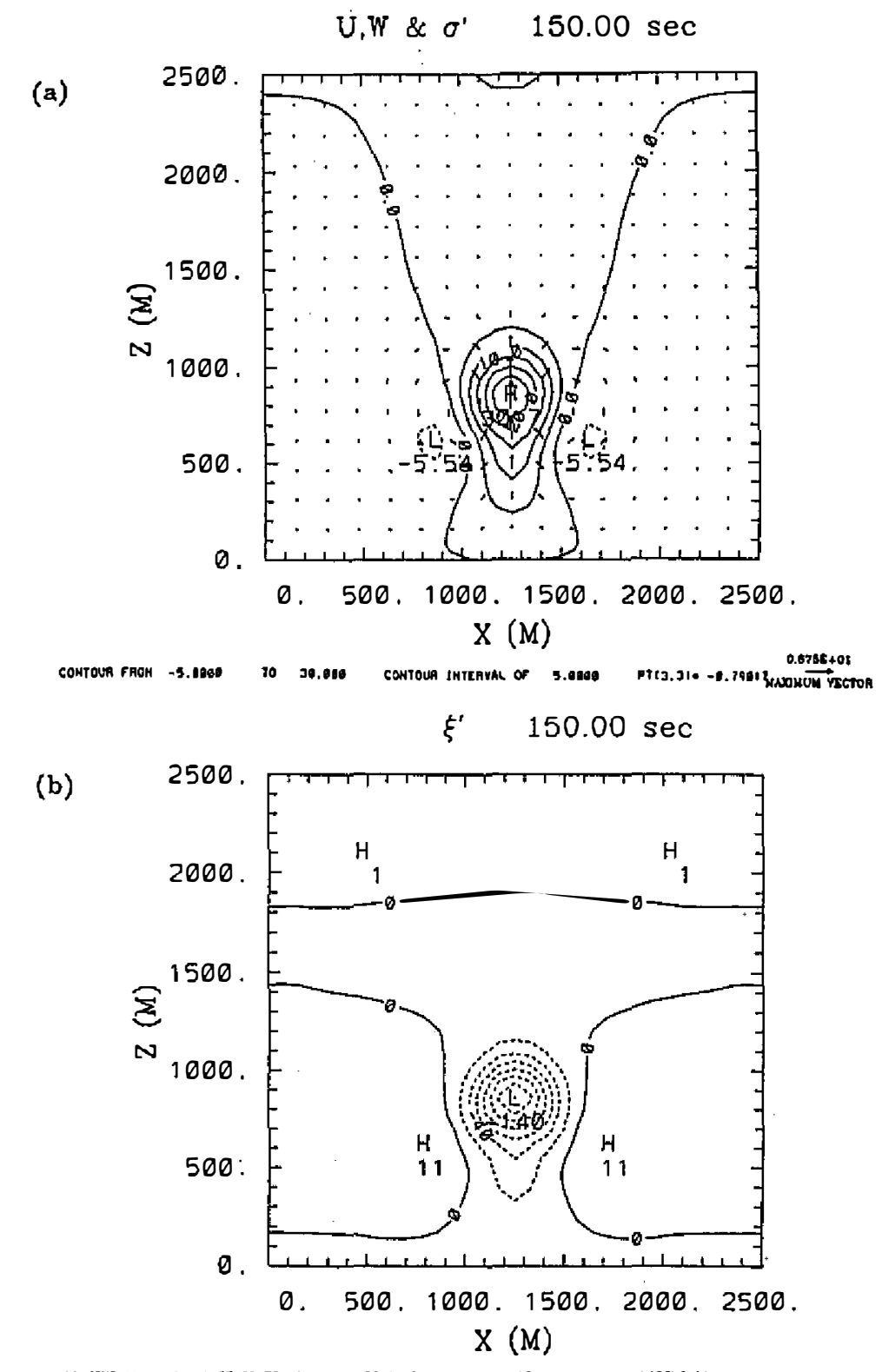

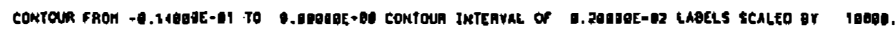

Fig. 12 The numerical results of EXP4 in $x-z$ domain at $t=150$ s for (a) the $U, W$ (the maximum vector is $6.75 \mathrm{~kg} \mathrm{~m}^{-2} \mathrm{~s}^{-1}$ ) and $\sigma^{\prime}$ (unit $\mathrm{J} \mathrm{m}^{-3} \mathrm{~K}^{-1}$, contour interval $5 \mathrm{~J} \mathrm{~m}^{-3} \mathrm{~K}^{-1}$ ), (b) $\xi^{\prime}$, (unit $10^{-4} \mathrm{~kg} \mathrm{~m}^{-3}$, contour interval $2 \times 10^{-3} \mathrm{~kg}$ $\mathrm{m}^{-3}$ ), (c) $p^{\prime}$ (unit $\mathrm{hPa}$, contour interval $0.1 \mathrm{hPa}$ ), (d) $T^{\prime}$ (unit $\mathrm{K}$, contour interval $0.3 \mathrm{~K}$ ), (e) $\eta^{\prime}$ (unit $10^{-5} \mathrm{~kg} \mathrm{~m}^{-3}$, contour interval $4 \times 10^{-4} \mathrm{~kg} \mathrm{~m}^{-3}$ ) and (f) liquid water density (unit $10^{-6} \mathrm{~kg} \mathrm{~m}^{-3}$, contour interval $3 \times 10^{-5} \mathrm{~kg}$ $\left.\mathrm{m}^{-3}\right)$. 


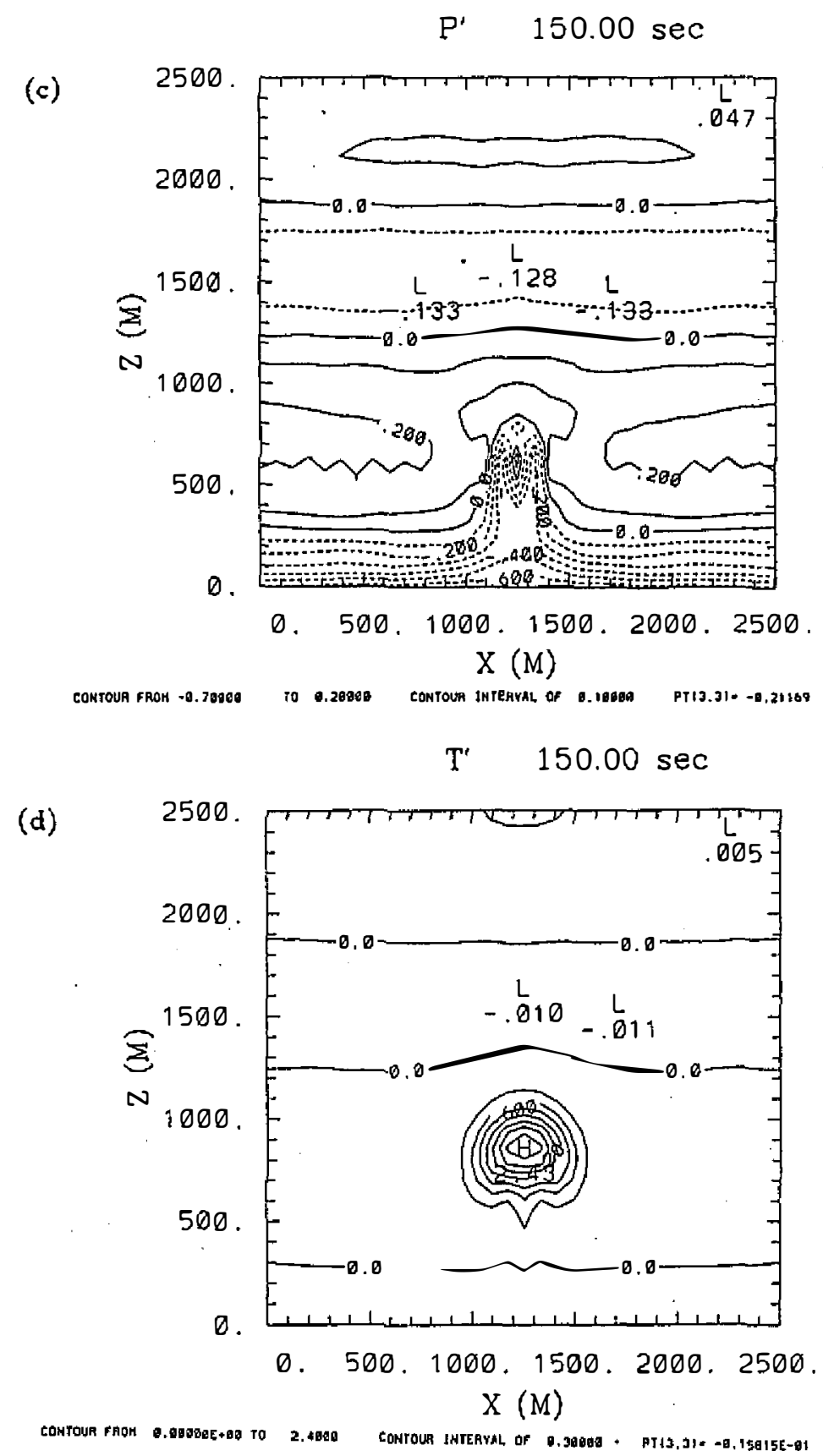

(Fig. 12. continued) 


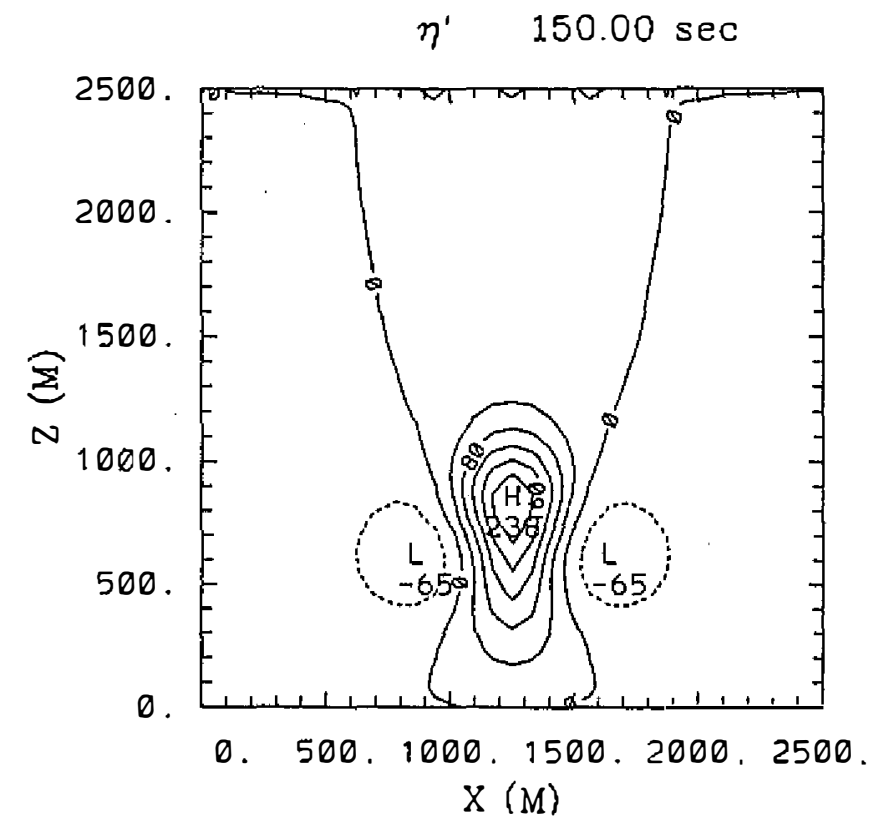

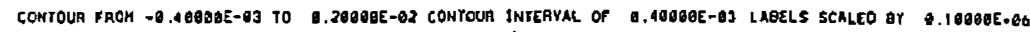
Liquid Water $150.00 \mathrm{sec}$

(f)

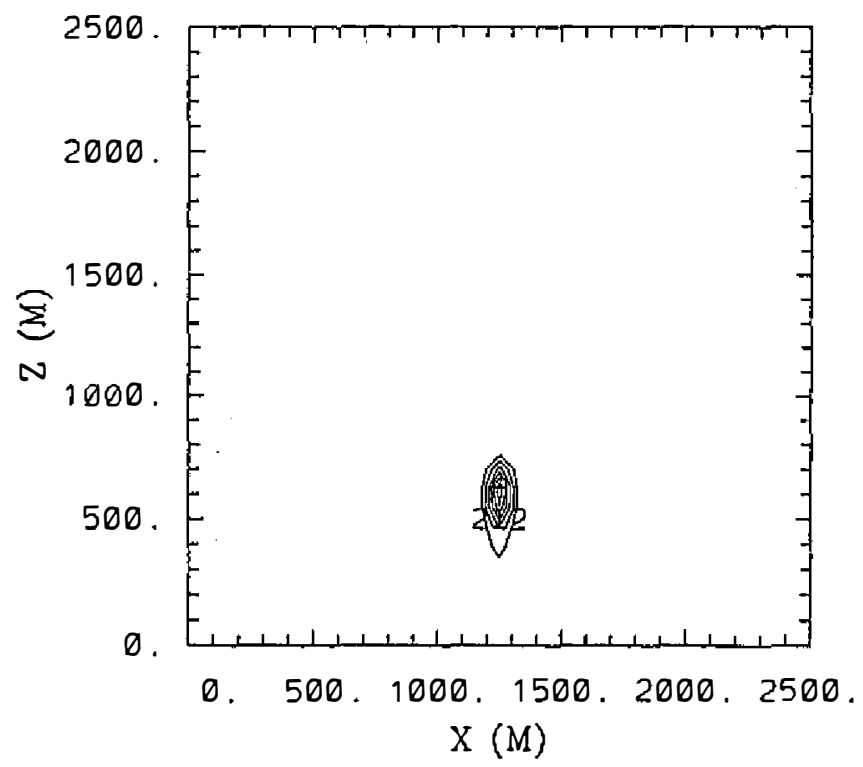

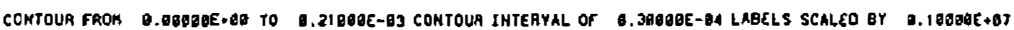

(Fig. 12. continued) 
$\mathrm{U}, W \& \sigma^{\prime} \quad 240.00 \mathrm{sec}$

(a)

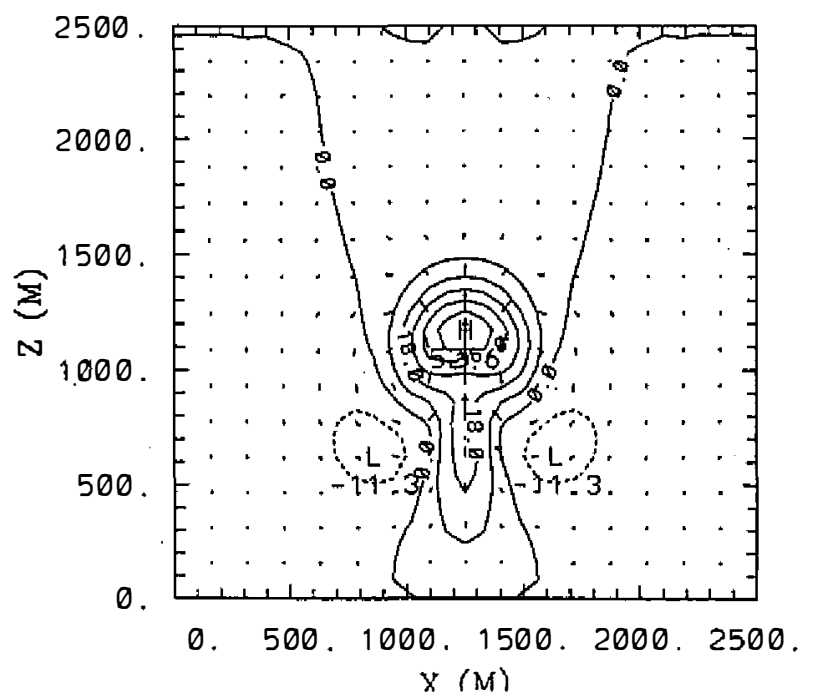

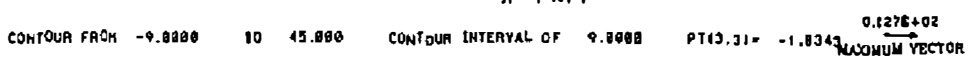

$\xi^{\prime} .240 .00 \mathrm{sec}$

(b)

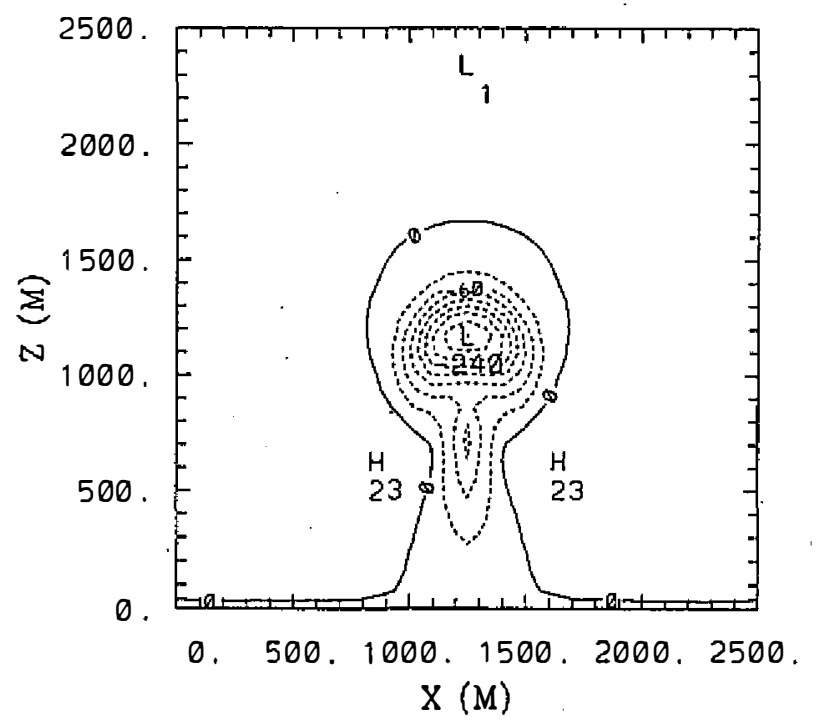

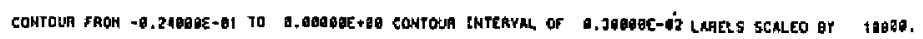

Fig. 13. Same as Fig. 12 except at $t=240$ s with (a) the $U, W$ (the maximum vector is $12.7 \mathrm{~kg} \mathrm{~m}^{-2} \mathrm{~s}^{-1}$ ) and $\sigma^{\prime}$ (unit $\mathrm{J} \mathrm{m}^{-3} \mathrm{~K}^{-1}$, contour interval $9 \mathrm{~J} \mathrm{~m}^{-3} \mathrm{~K}^{-1}$ ), (b) $\xi^{\prime}$, (unit $10^{-4} \mathrm{~kg} \mathrm{~m}^{-3}$, contour interval $3 \times 10^{-3} \mathrm{~kg} \mathrm{~m}^{-3}$ ), (c) $p^{\prime}$ (unit $\mathrm{hPa}$, contour interval $0.6 \mathrm{hPa}$ ), (d) $T^{\prime}$ (unit $\mathrm{K}$, contour interval $0.2 \mathrm{~K}$ ), (e) $\eta^{\prime}$ (unit $10^{-5} \mathrm{~kg} \mathrm{~m}^{-3}$, contour interval $9 \times 10^{-4} \mathrm{~kg} \mathrm{~m}^{-3}$ ) and (f) liquid water density (unit $10^{-5} \mathrm{~kg} \mathrm{~m}^{-3}$, contour interval $10^{-4} \mathrm{~kg} \mathrm{~m}^{-3}$ ). 


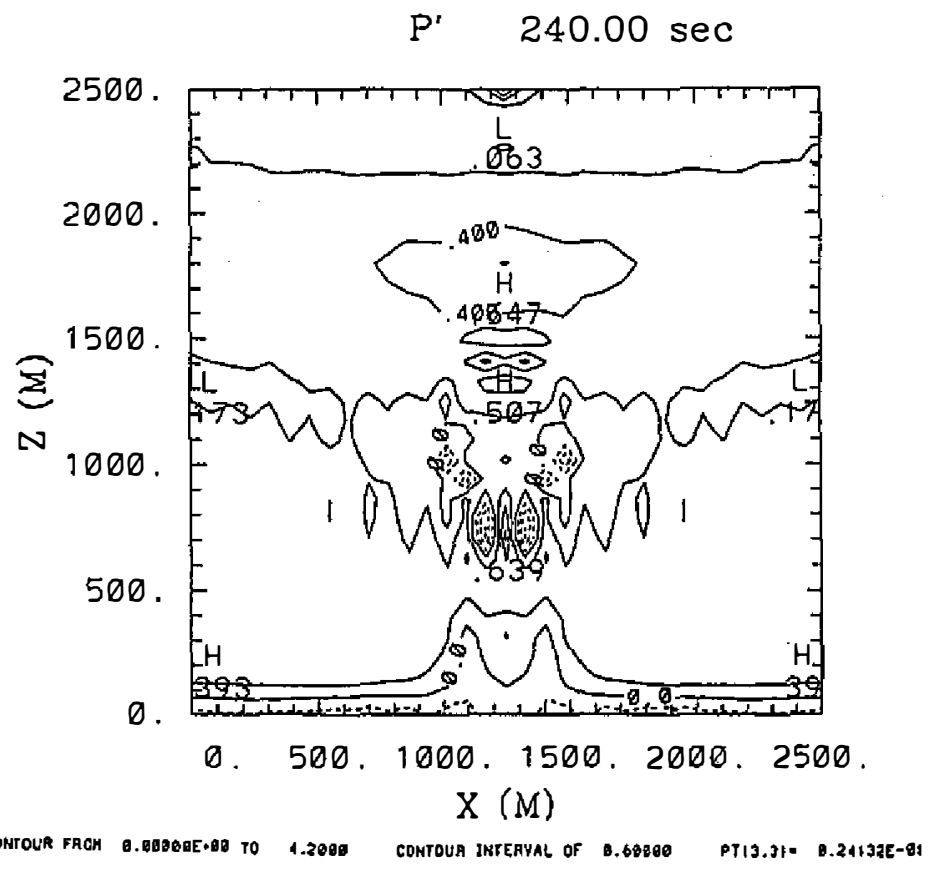

(c)

$\mathrm{T}^{\prime} \quad 240.00 \mathrm{sec}$

(d)

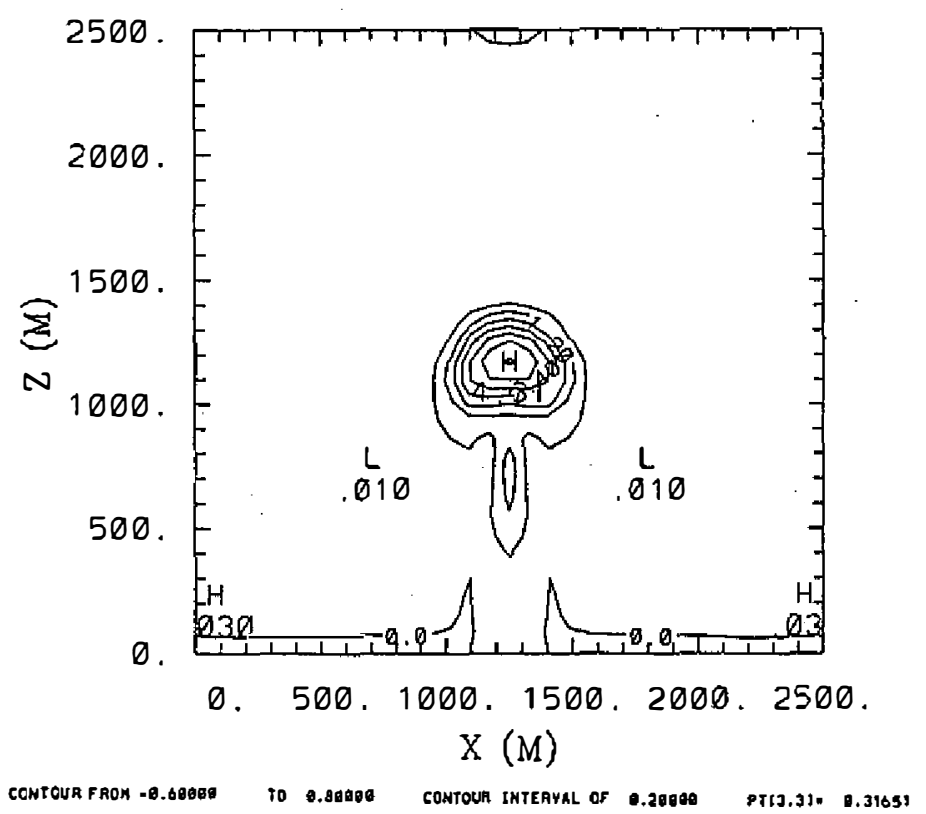

(Fig. 13. continued) 
$\eta^{\prime} \quad 240.00 \mathrm{sec}$

(e)

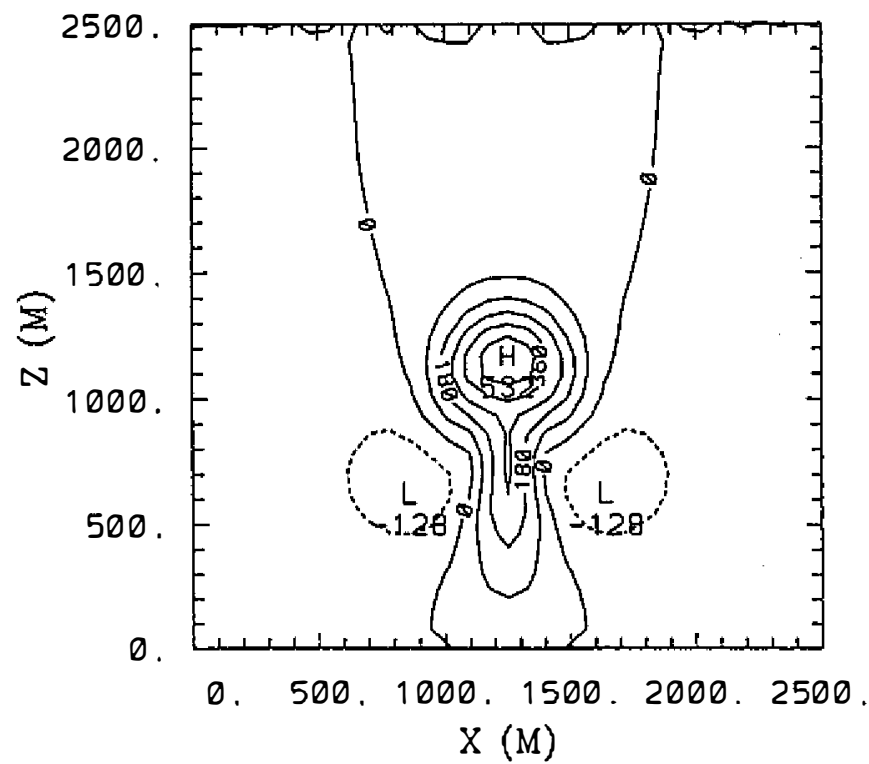

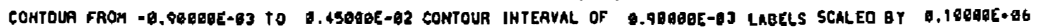

Liquid Water $240.00 \mathrm{sec}$

(f)

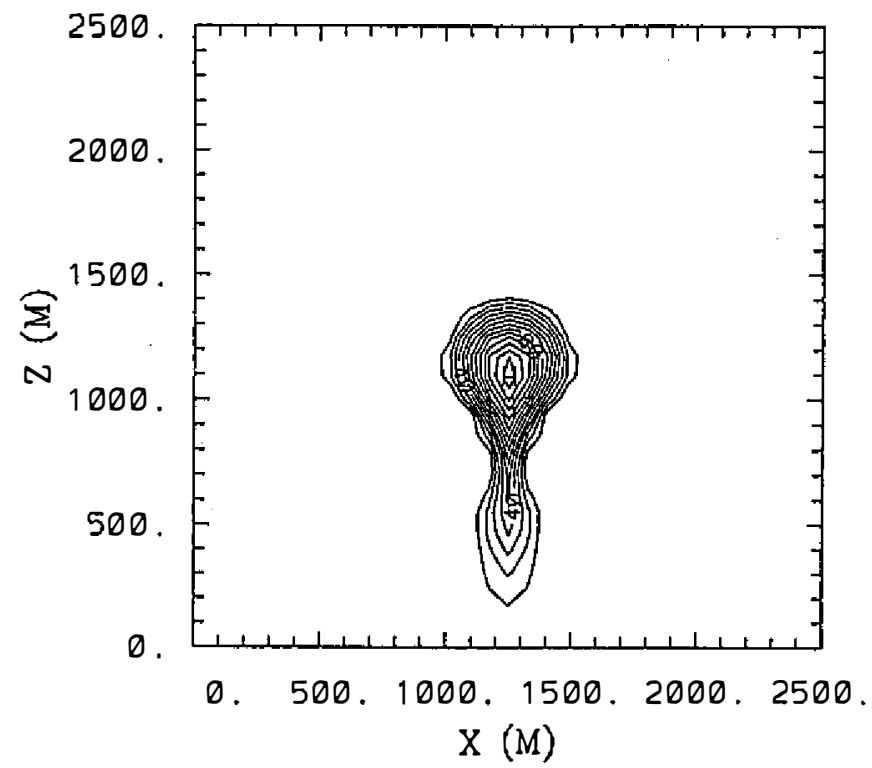

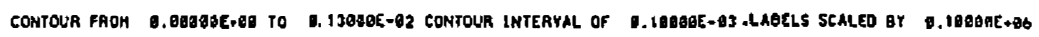

(Fig. 13. continued) 
latent heat effect. The general pattern of the high (low) pressure region above (below) the rising bubble in Fig. 13 is similar to the pattern of perturbation pressure in Figs. 9 and 10.

Our final experiment (EXP5) is related to condensation with a specified updraft. We consider the initial conditions of

$$
\begin{gathered}
\bar{r}_{t}=0.01275 \exp \left(-\frac{z}{1500}\right), \\
\bar{T}(z)=293.15-\frac{g}{C_{p d}} z,(K) \\
\bar{\xi}(z)=1.275\left(\frac{\bar{T}}{239.15}\right)^{\frac{c_{v d}}{R_{d}}} \exp \left[\frac{R_{v}}{R_{d}}\left(0.01275-\overline{r_{t}}\right)\right],\left(\mathrm{kgm}^{-3}\right) \\
\bar{\eta}=\overline{r_{t} \bar{\xi}},\left(\mathrm{kgm}^{-3}\right) \\
U(x, z)=5 \times \sin \phi \times \sin \left(\frac{z}{2500} \pi\right) \exp \left[-\left(\frac{x-x_{0}}{200}\right)^{2}\right],\left(\mathrm{kgm}^{-3} \mathrm{~ms}^{-1}\right) \\
W(x, z)=5 \times \cos \phi \times \sin \left(\frac{z}{2500} \pi\right) \exp \left[-\left(\frac{x-x_{0}}{200}\right)^{2}\right],\left(k g m^{-3} \mathrm{~ms}^{-1}\right)
\end{gathered}
$$

where

$$
x_{0}=1250+(z-1250) \tan \phi \text {, }
$$

and where $\phi$ is the tilt angle from the vertical.

Figure 14 presents the time series of the maximum value of $\xi^{\prime}, W, \eta_{c}$ and $T^{\prime}$ for the $\phi=$ 0 and $\phi=\pi / 6$ cases. The results from Fig. 14 are expected in that the tilted updraft $(\phi=\pi \prime$ 6) produced less condensed water, $T^{\prime}$, and $W$ in the later stage. The numerical results of the perturbation fields in the $x-z$ domain at $t=240$ s with the initial momentum density condition computed with $\phi=\pi / 6$ are presented in Fig. 15. Because of the vertical gradient of $\eta, \sigma$ and $\xi$ in the initial conditions, there are positive anomalies of $\eta$ and $\sigma$ which are brought up by the updraft. In addition, the positive anomaly of $T^{\prime}$ and the negative anomaly of $\xi^{\prime}$ are associated with the condensation of liquid water. Rotunno et al. (1988) viewed the no tilted updraft situation as the "optimal state" for the squall line. Moreover, they argued that the tilted updraft may be stemmed from the imbalance of the vorticity across the low level gust front. On the other hand, Seitter and Kuo (1983) argued the tilted updraft may be stemmed from the liquid water loading effect in the updraft/downdraft interface. The study of tilted updraft is of fundamental importance in understanding long-lived mesoscale convection. EXP5 suggests that our 
(a)

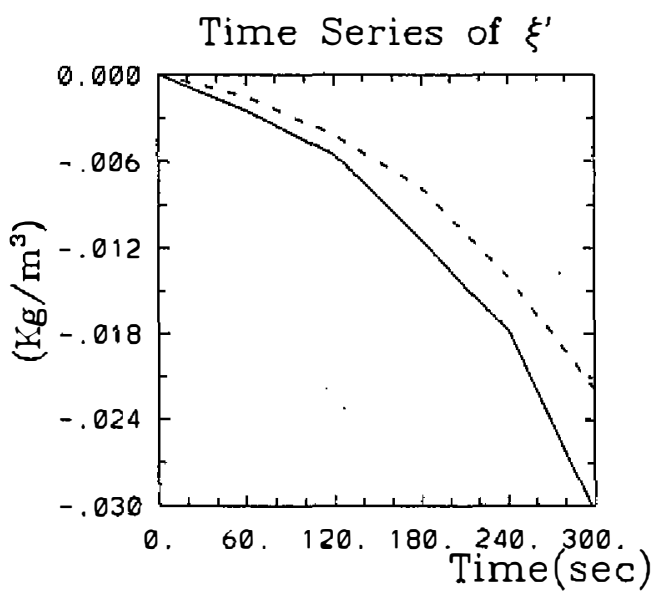

Time Series of Max W

(b)

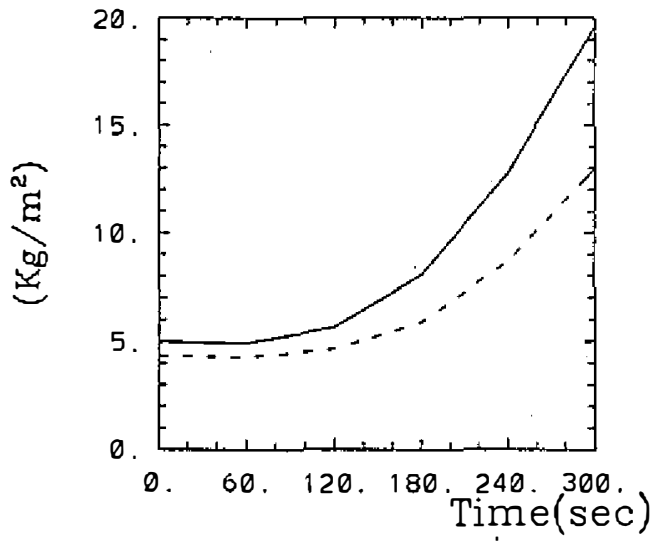

Time Series of Liquid Water

(c)

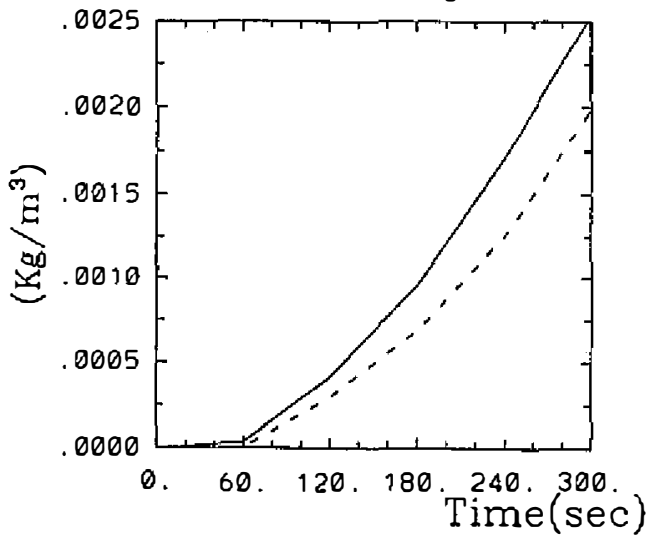

Time Series of $T^{\prime}$

(d)

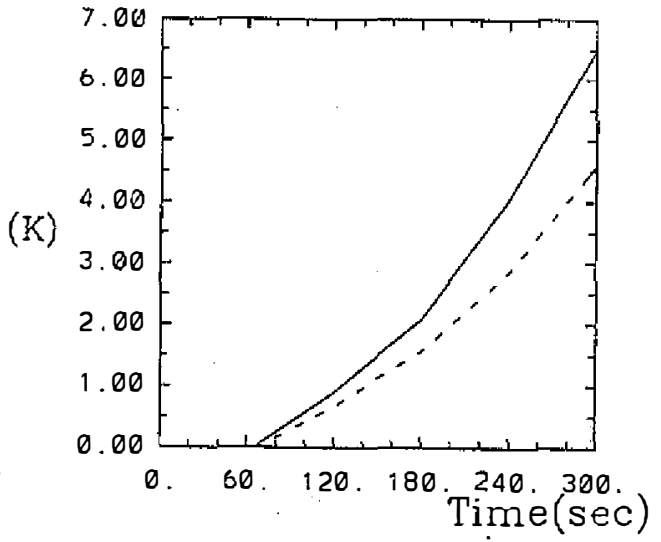

Fig. 14. The time series in EXP5 for (a) maximum $\xi^{\prime}$ (unit $\mathrm{kg} \mathrm{m}^{-3}$ ), (b) maximum vertical momentum density $W$ (unit $\mathrm{kg} \mathrm{m}^{-2} \mathrm{~s}^{-1}$ ), (c) maximum liquid water density (unit $\mathrm{kg} \mathrm{m}^{-3}$ ) and (d) maximum $T^{\prime}$ (unit $K$ ). The dashed curve is for the case of updraft with tilt angle $\phi=\pi / 6$ and the solid curve is for case of $\phi=0$.

model is capable of simulating condensation associated with a tilted updraft.

\section{CONCLUDING REMARKS}

Some points of the model worth noting are as follows.

1. The temperature and pressure are diagnostically determined from thermodynamics.

2 . There is no need to predict water vapor and condensate separately; rather, they are diagnostically separated from the predicted total airborne water. 


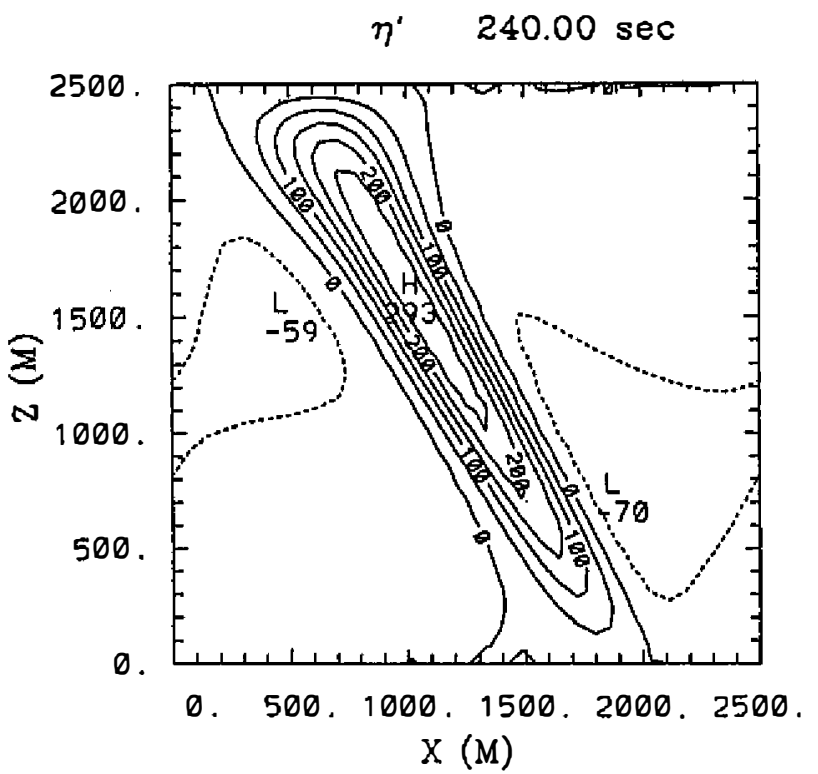

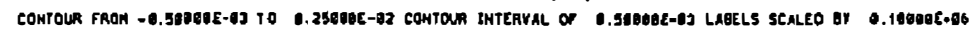

(b)

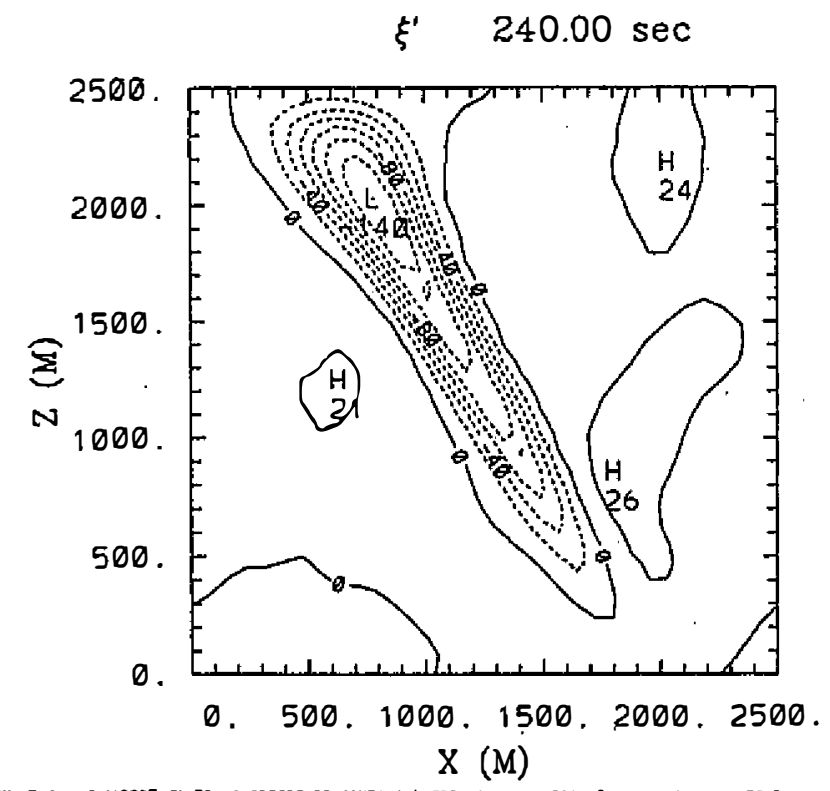

Fig. 15. The numerical results of the perturbation fields in $x-z$ domain at $t=240 \mathrm{~s}$ with the initial momentum density condition computed with $\phi=\pi / 6$ for (a) $\eta^{\prime}$ (unit $10^{-5} \mathrm{~kg} \mathrm{~m}^{-3}$, contour interval $5 \times 10^{-4} \mathrm{~kg} \mathrm{~m}^{-3}$ ), (b) $\xi^{\prime}$, (unit $10^{-4} \mathrm{~kg} \mathrm{~m}^{-3}$, contour interval $2 \times 10^{-3} \mathrm{~kg} \mathrm{~m}^{-3}$ ), (c) liquid water density (unit $10^{-5} \mathrm{~kg} \mathrm{~m}^{-3}$, contour interval $10^{-4} \mathrm{~kg} \mathrm{~m}^{-3}$ ) and (d) $T^{\prime}$ (unit $\mathrm{K}$, contour interval $0.4 \mathrm{~K}$ ). 
Hung-Chi Kuo \& Chao-Tzuen Cheng

(c)

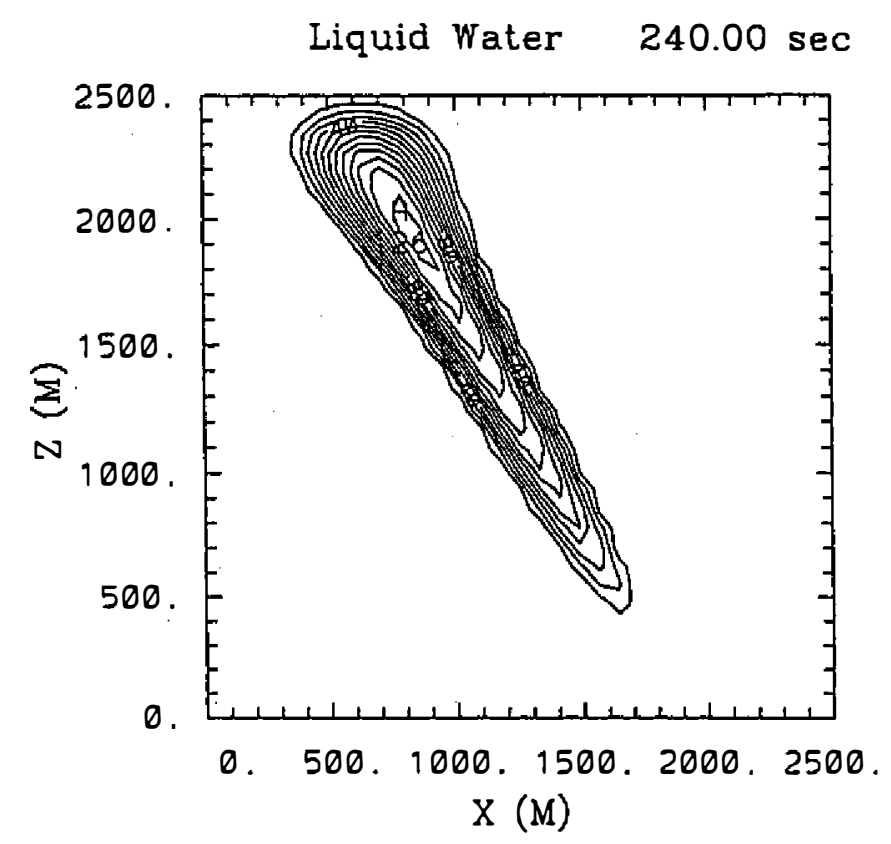

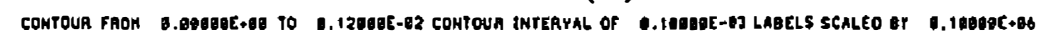

(d)

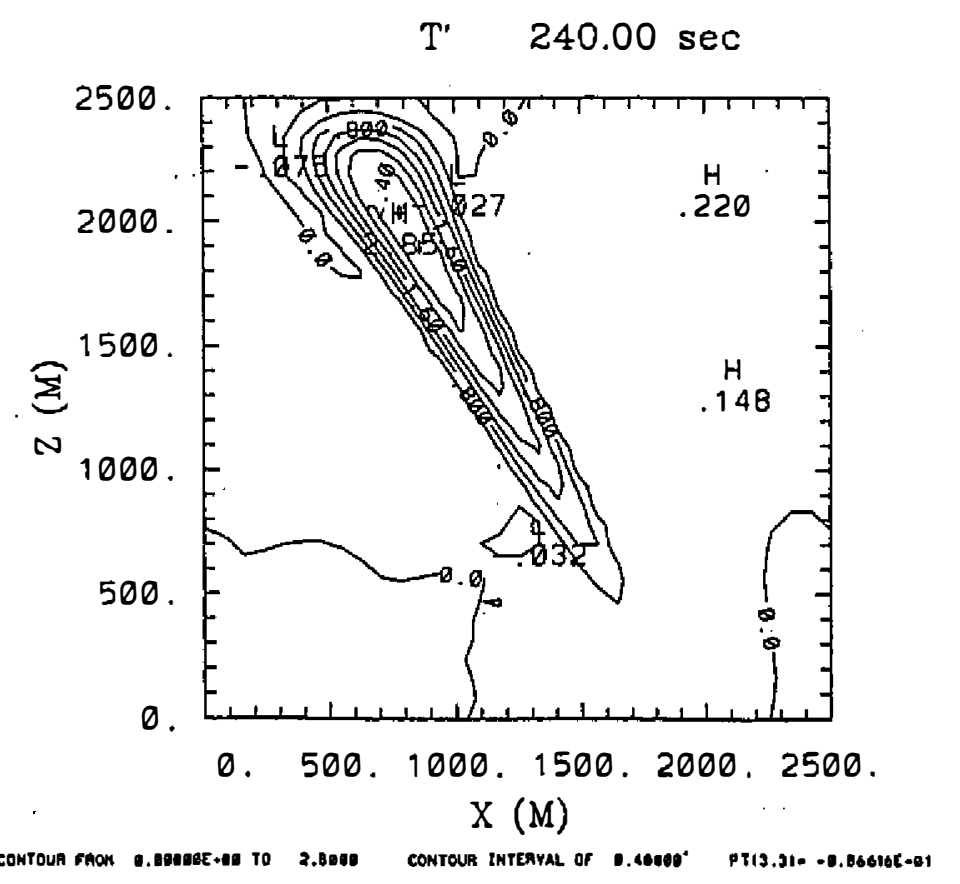

(Fig. 15. continued) 
3. There is a modular separation of dynamics and thermodynamics; the link between dynamics and thermodynamics is through the pressure gradient force. When the hydrostatic approximation is made, the only change in the model is how the vertical motion is computed.

4. The discontinuity in thermodynamics due to phase change can be modified to a "gradual saturation" in order to make the moist thermodynamics match the spatial resolution of the model.

We have not included the ice phase or the precipitating processes in our experiments. As discussed in Ooyama (1990), the ice phase can be handled by a hypothetical single phase condensate that behaves like liquids at warm temperatures and like ice at cold temperatures, with a gradual transition at intermediate temperatures. Ooyama (1995) tested warm rain microphysics in the formation of squall lines. One advantage of Ooyama's approach is that it allows us to have a detailed, fine resolution, nonhydrostatic cloud model and a coarse resolution, hydrostatic (GCM-like) model which can be run side by side with identical moist thermodynamics. The height coordinate of the nonhydrostatic model can also extend into the hydrostatic regime. The only differences between the two models are spatial resolution and the way vertical motion is computed. This allows us to analyze in detail what physics is lost (and hence needs to be parameterized) as model resolution coarsens and nonhydrostatic dynamics is replaced by hydrostatic dynamics.

The work presented here is considered a building block for the theoretical studies which employ both least assumptions in moist physics and a very accurate numerical solution method. We are currently improving the efficiency of time discretization. The convection model will be used in the future to study the interaction of radiative, convective, and drizzle effects.

Acknowledgments This research was supported by Grants NSC87-2111-M002-005AP1 from the National Research Council of Taiwan. The work represents a portion of the second author's master thesis at National Taiwan University. We like to thank Dr. W. H. Schubert for useful comments throughout the study: We also like to thank the reviewers for their very careful reviews.

\section{Appendix A}

\section{Diagnostic Equations for Vertical Motion}

We consider the diagnostic equations for vertical motion in two forms. The main results are in (A.6) and (A.11). The first one is the Richardson vertical motion equation in differential form. We start with the dry equation of state $p=\rho R T$ (here $R=R_{d}, \rho=\xi, c_{p}=c_{p d}$ and $c_{\nu}=c_{v d}$ ) to get

$$
\frac{d \ln p}{d t}=\frac{d \ln \rho}{d t}+\frac{d \ln \gamma}{d t}
$$

From the continuity equation

$$
\frac{d \ln \rho}{d t}=-\frac{\partial v_{i}}{\partial x_{i}}-\frac{\partial w}{\partial z}, \quad \mathrm{i}=1,2
$$


and the first law of thermodynamics

$$
\frac{d \ln T}{d t}=\frac{R}{c_{p}} \frac{d \ln p}{d t}+\frac{\dot{Q}}{c_{p} T},
$$

we obtain

$$
\frac{\partial p}{\partial t}=-v_{i} \frac{\partial p}{\partial x_{i}}-w \frac{\partial p}{\partial z}-\frac{c_{p}}{c_{v}} p\left(\frac{\partial v_{i}}{\partial x_{i}}+\frac{\partial w}{\partial z}\right)+p \frac{\dot{Q}}{c_{v} T}
$$

Note that $\left(c_{p} / c_{v}\right) p$ in (A.4) can be written as $\left(c_{p} / c_{v}\right) p=\left(c_{p} / c_{v}\right) \rho R T=\rho C^{2}$ where $C^{2}$ is the Laplace adiabatic sound speed.

With the help of the time differentiated hydrostatic equation

$$
\frac{\partial^{2} p}{\partial t \partial z}=-\frac{\partial \rho}{\partial t} g=\left(\frac{\partial\left(\rho v_{i}\right)}{\partial x_{i}}+\frac{\partial(\rho w)}{\partial z}\right) g
$$

(A.4) can now be written as

$$
\frac{\partial}{\partial z}\left(\rho C^{2} \frac{\partial w}{\partial z}\right)=-\frac{\partial}{\partial z}\left(v_{i} \frac{\partial p}{\partial x_{i}}+\rho C^{2} \frac{\partial v_{i}}{\partial x_{i}}-p \frac{\dot{Q}}{c_{v} T}\right)-\frac{\partial \rho v_{i}}{\partial x_{i}} g-\frac{\partial}{\partial z}\left[w\left(\rho g+\frac{\partial p}{\partial z}\right)\right]
$$

or

$$
\frac{\partial}{\partial z}\left(\rho C^{2} \frac{\partial w}{\partial z}\right)=-\frac{\partial}{\partial z}\left(v_{i} \frac{\partial p}{\partial x_{i}}+\rho C^{2} \frac{\partial v_{i}}{\partial x_{i}}-p \frac{\dot{Q}}{c_{v} T}\right)-\frac{\partial \rho v_{i}}{\partial x_{i}} g
$$

Equation (A.6) is the Richardson vertical motion equation in differential form.

We now derive the vertical equation based on Ooyama (1990) moist thermodynamics in $\sigma-z$ vertical coordinate. The derivation is similar to that in the height coordinate by DeMaria (1995). The $\sigma-z$ coordinate is defined by

$$
z^{\prime}=H\left(\frac{z-z_{s}}{H-z_{s}}\right)
$$

where $z_{s}$ is the topographic height. The conservation equations after the coordinate transform can be written as

$$
\frac{\partial \sigma^{\prime}}{\partial t}+\frac{\partial\left(\sigma^{\prime} v_{i}\right)}{\partial x_{i}^{\prime}}+\frac{\partial\left(\sigma^{\prime} w^{\prime}\right)}{\partial z^{\prime}}=Q_{\sigma^{\prime}}
$$




$$
\begin{gathered}
\frac{\partial \xi^{\prime}}{\partial t}+\frac{\partial\left(\xi^{\prime} v_{i}\right)}{\partial x_{i}^{\prime}}+\frac{\partial\left(\xi^{\prime} w^{\prime}\right)}{\partial z^{\prime}}=0 \\
\frac{\partial \eta^{\prime}}{\partial t}+\frac{\partial\left(\eta^{\prime} v_{i}\right)}{\partial x_{i}^{\prime}}+\frac{\partial\left(\eta^{\prime} w^{\prime}\right)}{\partial z^{\prime}}=Q_{\eta^{\prime}}
\end{gathered}
$$

and the hydrostatic equation can be written as

$$
\frac{\partial p}{\partial z^{\prime}}=-\rho^{\prime} g
$$

where $\xi^{\prime}=\xi\left(H-z_{s}\right) / H, \eta^{\prime}=\eta\left(H-z_{s}\right) / H, \rho^{\prime}=\eta^{\prime}+\xi^{\prime}, \sigma^{\prime}=\sigma\left(H-z_{s}\right) / H$, and

$$
w^{\prime}=\frac{H}{H-z_{s}}\left[w+\left(\frac{z^{\prime}}{H}-1\right) v_{i} \frac{\partial z_{s}}{\partial x_{i}^{\prime}}\right] .
$$

Similar to the definition of adiabatic sound speed in (2.10), we define $C^{2}$ by

$$
C^{2}=\frac{\partial p}{\partial \rho^{\prime}}+\frac{\sigma^{\prime}}{\rho^{\prime}} \frac{\partial p}{\partial \sigma^{\prime}}
$$

By taking $\partial / \partial t$ of the hydrostatic equation to give

$$
\frac{\partial}{\partial z^{\prime}}\left(\frac{\partial p}{\partial t}\right)=-\frac{\partial \rho^{\prime}}{\partial t} g
$$

Eliminating $\partial p / \partial t$ in (A.10) and eliminating time derivatives with (A.7) yields the Ooyama vertical diagnostic equation in $\sigma-z$ vertical coordinate

$$
\frac{\partial}{\partial z^{\prime}}\left(\rho C^{\prime 2} \frac{\partial w^{\prime}}{\partial z^{\prime}}\right)=-\frac{\partial}{\partial z^{\prime}}\left(v_{i} \frac{\partial p}{\partial x_{i}^{\prime}}+\rho^{\prime} C^{\prime 2} \frac{\partial v_{i}}{\partial x_{i}^{\prime}}\right)-\frac{\partial \rho^{\prime} v_{i}}{\partial x_{i}^{\prime}} g+\frac{\partial}{\partial z^{\prime}}\left(\frac{\partial p}{\partial \sigma^{\prime}} Q_{\sigma^{\prime}}+\frac{\partial p}{\partial \rho^{\prime}} Q_{\eta^{\prime}}\right)+g Q_{\eta^{\prime}}
$$




\section{Appendix B}

\section{Derivation of pressure coefficients}

We consider both saturated and unsaturated states of the air in the derivation of pressure coefficients. The derived pressure coefficients are in (B.4) and (B.8). The variables take the usual meteorological meanings.

For the unsaturated air we start with the equation of state $p=\left(\xi R_{a}+\eta R_{v}\right) T$ and obtain

$$
\begin{gathered}
P_{\sigma}^{(1)}=\frac{\partial p}{\partial \sigma}=\left(\xi R_{a}+\eta R_{v}\right) \frac{\partial T}{\partial \sigma} \\
P_{\xi}^{(1)}=\frac{\partial p}{\partial \xi}=\left(\xi R_{a}+\eta R_{v}\right) \frac{\partial T}{\partial \xi}+R_{a} T \\
P_{\eta}^{(1)}=\frac{\partial p}{\partial \eta}=\left(\xi R_{a}+\eta R_{v}\right) \frac{\partial T}{\partial \eta}+R_{v} T
\end{gathered}
$$

The entropy density for the unsaturated air is

$$
\sigma=\left(\xi c_{v a}+\eta c_{v v}\right) \ln \frac{T}{T_{0}}+\eta \Lambda_{0}-\xi R_{a} \ln \frac{\xi}{\xi_{0}}-\eta R_{v} \ln \frac{\eta}{\eta_{0}^{*}}
$$

By using $\partial \sigma / \partial \sigma=1, \partial \sigma / \xi=0$, and $\partial \sigma / \partial \eta=0$, we have

$$
\begin{gathered}
\frac{\partial T}{\partial \sigma}=T\left(\xi_{c_{v a}}+\eta c_{v v}\right)^{-1} \\
\frac{\partial T}{\partial \xi}=T\left(\xi_{c_{v a}}+\eta c_{v v}\right)^{-1}\left(R_{u}+R_{u} \ln \frac{\xi}{\xi_{0}}-c_{v a} \ln \frac{T}{T_{0}}\right) \\
\frac{\partial T}{\partial \eta}=T\left(\xi_{c_{v a}}+\eta c_{v v}\right)^{-1}\left(R_{v}+R_{v} \ln \frac{\eta}{\eta_{0}^{*}}-c_{v v} \ln \frac{T}{T_{0}}\right)-\Lambda_{0}
\end{gathered}
$$

From (B.1) and (B.3) we get pressure coefficients for the unsaturated air 


$$
\begin{gathered}
P_{\sigma}^{(1)}=\left(\xi c_{v a}+\eta c_{v v}\right)^{-1}\left(\xi R_{u}+\eta R_{v}\right) T \\
P_{\xi}^{(1)}=R_{u} T+\left[R_{u}\left(1+\ln \frac{\xi}{\xi_{0}}\right)-c_{v a} \ln \frac{T}{T_{0}}\right] P_{\sigma}^{(1)}, \\
P_{\eta}^{(1)}=R_{v} T+\left[R_{v}\left(1+\ln \frac{\eta}{\eta_{0}^{*}}\right)-c_{v v} \ln \frac{T}{T_{0}}-\Lambda_{0}\right] P_{\sigma}^{(1)} .
\end{gathered}
$$

For the saturated air we start with the equation of state $p=\left(\xi R_{a}+\eta^{*} R_{v}\right) T=\xi R_{a} T+E(T)$ and obtain

$$
\begin{gathered}
P_{\sigma}^{(2)}=\frac{\partial p}{\partial \sigma}=\xi R_{a} \frac{\partial T}{\partial \sigma}+\frac{\partial E(T)}{\partial T} \frac{\partial T}{\partial \sigma}=\left[\xi R_{a}+D(T)\right] \frac{\partial T}{\partial \sigma} \\
P_{\xi}^{(2)}=\frac{\partial p}{\partial \xi}=R_{a} T+\xi R_{a} \frac{\partial T}{\partial \xi}+\frac{\partial E(T)}{\partial T} \frac{\partial T}{\partial \xi}=R_{a} T+\left[\xi R_{a}+D(T)\right] \frac{\partial T}{\partial \xi} \\
P_{\eta}^{(2)}=\frac{\partial p}{\partial \eta}=\xi R_{a} \frac{\partial T}{\partial \eta}+\frac{\partial E(T)}{\partial T} \frac{\partial T}{\partial \eta}=\left[\xi_{a}+D(T)\right] \frac{\partial T}{\partial \eta}
\end{gathered}
$$

The entropy density for the saturated air is

$$
\sigma=\eta c_{v a} \ln \frac{T}{T_{0}}-\xi R_{a} \ln \frac{\xi}{\xi_{0}}+\eta C(T)+D(T)
$$

Similar to the derivation of (B.3), we derived

$$
\begin{gathered}
\frac{\partial T}{\partial \sigma}=\left(\frac{\xi c_{v a}}{T}+\eta \frac{d C}{d T}+\frac{d D}{d T}\right)^{-1}, \\
\frac{\partial T}{\partial \xi}=\left[R_{a}\left(1+\ln \frac{\xi}{\xi_{0}}\right)-c_{v v} \ln \frac{T}{T_{0}}\right]\left(\frac{\xi c_{v a}}{T}+\eta \frac{d C}{d T}+\frac{d D}{d T}\right)^{-1}, \\
\frac{\partial T}{\partial \sigma}=-C(T)\left(\frac{\xi c_{v a}}{T}+\eta \frac{d C}{d T}+\frac{d D}{d T}\right)^{-1} .
\end{gathered}
$$


From (B.5) and (B.7) we get pressure coefficients for the saturated air

$$
\begin{gathered}
P_{\sigma}^{(2)}=\left[\xi R_{a}+D(T)\right]\left(\frac{\xi c_{v a}}{T}+\eta \frac{d C}{d T}+\frac{d D}{d T}\right)^{-1}, \\
P_{\xi}^{(2)}=R_{a} T+\left[R_{a}\left(1+\ln \frac{\xi}{\xi_{0}}\right)-c_{v a} \ln \frac{T}{T_{0}}\right] P_{\sigma}^{(2)}, \\
P_{\eta}^{(2)}=-C(T) P_{\sigma}^{(2)},
\end{gathered}
$$

where

$$
\begin{gathered}
C(T)=c_{v v} \ln \frac{T}{T_{0}}-R_{\nu} \ln \frac{\eta^{*}}{\eta_{0}^{*}}-\Lambda(T)+\Lambda\left(T_{0}\right), \\
D(T)=\eta^{*} \Lambda(T)=\frac{d E(T)}{d T} \\
\Lambda(T)=\frac{l_{c}(T)}{T}=R_{v} T \frac{d \ln E(T)}{d T}
\end{gathered}
$$

and

$$
\eta^{*}=\frac{E(T)}{R_{v} T}
$$

\section{REFERENCES}

Bannon, P. R., 1995: Hydrostatic adjustment: Lamb's problem. J. Atmos. Sci., 52, 1743-1752.

Duffy, D. G., 1997: Hydrostatic adjustment in nonhydrostatic, compressible mesoscale models. Mon. Wea. Rev., 125, 3357-3367.

DeMaria, M., 1995: Evaluation of a hydrostatic, height-coordinate formulation of the primitive equations for atmospheric modeling. Mon. Wea. Rev., 123, 3576-3589.

Fulton, S. R., and W. H. Schubert, 1987: Chebyshev spectral methods for limited-area models. Part I: Model problem analysis. Mon. Wea. Rev., 115, 1940-1953.

Fulton, S. R., and W. H. Schubert, 1987: Chebyshev spectral methods for limited-area models. Part II: Shallow water model. Mon. Wea. Rev., 115, 1954-1965.

Gottlieb, D., and S. A. Orszag, 1977: Numerical analysis of spectral methods. NSF-CBMS Monograph No. 26, NTIS No. AD-A056 922, Soc. Ind. and Appl. Math., Philadelphia, $172 \mathrm{pp}$. 
Kuo, H.-C., and W. H. Schubert, 1988: Stability of cloud-topped boundary layers. Quart. J. R. Meteor. Soc., 114, 887-916.

Ogura, Y., and N. A. Phillips, 1962: Scale analysis of deep and shallow convection in atmosphere. J. Atmos. Sci., 19, 173-179.

Ooyama, K. V., 1990: A thermodynamic foundation for modeling the moist atmosphere. J. Atmos. Sci., 47, 2580-2593.

Ooyama, K. V., 1995: A thermodynamic foundation for modeling the moist atmosphere, Part II: Tests of microphysics in the formation of squall lines. Preprints, 21st Conference on Hurricanes and Tropical Meteorology. Amer. Meteor. Soc., Boston, 531-532.

Rotunno, R., J. B. Klemp, and M. L. Weisman, 1988: A theory for strong, long lived squall lines. J. Atmos. Sci., 45, 463-485.

Seitter, K., and H. L. Kuo, 1983: The dynamical structure of squall-line type thunderstorms. J. Atmos. Sci., 40, 2831-2854. 